\title{
Stealth nanotubes: strategies of shielding carbon nanotubes to evade opsonization and improve biodistribution
}

This article was published in the following Dove Press journal:

International Journal of Nanomedicine

6 May 2014

Number of times this article has been viewed

\author{
Nalinikanth Kotagiri ${ }^{1-4}$ \\ Jin-Woo Kim ${ }^{1-3}$ \\ 'Bio/Nano Technology Laboratory, \\ Institute for Nanoscience and \\ Engineering, ${ }^{2}$ Department of Biological \\ and Agricultural Engineering, ${ }^{3} \mathrm{Cell}$ and \\ Molecular Biology Graduate Program, \\ University of Arkansas, Fayetteville, \\ AR, USA; ${ }^{4}$ Optical Radiology \\ Laboratory, Department of Radiology, \\ Washington University School of \\ Medicine, St Louis, MO, USA
}

\begin{abstract}
Carbon nanotubes (CNTs) have recently been in the limelight for their potential role in disease diagnostics and therapeutics, as well as in tissue engineering. Before these medical applications can be realized, there is a need to address issues like opsonization, phagocytosis by macrophages, and sequestration to the liver and spleen for eventual elimination from the body; along with equally important issues such as aqueous solubility, dispersion, biocompatibility, and biofunctionalization. CNTs have not been shown to be able to evade such biological obstacles, which include their nonspecific attachments to cells and other biological components in the bloodstream, before reaching target tissues and cells in vivo. This will eventually determine their longevity in circulation and clearance rate from the body. This review article discusses the current status, challenges, practical strategies, and implementations of coating CNTs with biocompatible and opsonin-resistant moieties, rendering CNTs transparent to opsonins and deceiving the innate immune response to make believe that the CNTs are not foreign. A holistic approach to the development of such "stealth" CNTs is presented, which encompasses not only several biophysicochemical factors that are not limited to surface treatment of CNTs, but also extraneous biological factors such as the protein corona formation that inevitably controls the in vivo fate of the particles. This review also discusses the present and potential applications, along with the future directions, of CNTs and their hybrid-based nanotheranostic agents for multiplex, multimodal molecular imaging and therapy, as well as in other applications, such as drug delivery and tissue engineering.
\end{abstract}

Keywords: opsonins, macrophage, in vivo biocompatibility, near-infrared contrast nanoagents, nanotheranostics, nanomedicine

\section{Introduction}

The last decade has seen the emergence of a new field of medicine, aptly termed "nanomedicine", which utilizes nanoparticles (NPs) and similar materials to diagnose and treat afflictions of the human body. It is logical that an intravenous route will be the preferential mode of administering the NPs. Also, given that most end capillaries are not more than 5-6 $\mu \mathrm{m}$ in diameter, it can be safely assumed that NPs are preferred over microparticles to prevent aggregation and clogging of end vessels. The uniqueness of NPs comes from their dimensions (ie, 1-100 nm), which are larger than atoms and smaller than cells. ${ }^{1}$ This allows for engineered NPs to be designed in such a way that they tag specific cells, such as tumor cells, and enable monitoring and destruction of the respective cell type. Innovative approaches have been proposed to design and construct NPs and their architectures with desirable size, shape and functionality, including those based on the programmable self-assembly approach. ${ }^{2,3}$ Such
Correspondence: Jin-Woo Kim

gical Engineering, Biomedica

Engineering, 203 White Engineering Hall,

University of Arkansas, Fayetteville

AR 7270I, USA

Tel + I 479575235 I

Fax + I 4795752846

Email jwkim@uark.edu
International Journal of Nanomedicine 2014:9 (Suppl I) 85-105 
specificity and precision in targeting individual cells is an encouraging departure from drugs that systemically target cells (even normal cells), leading to undesired side effects. ${ }^{4}$ Furthermore, the availability of many unique NPs with their characteristic physical, chemical, and optical properties, such as photostable fluorescence, multiple conjugation sites for ligands, and tunable optical responsiveness to ultraviolet, near infrared (NIR), and infrared radiations, etc, is advantageous for developing highly versatile targeting platforms. ${ }^{2,3,5,6}$

Research groups that have been interested in the biomedical applications of these NPs have the luxury to choose from these signature properties to suit their individual needs, and to tune the NPs accordingly for their desired effects. One of the nanomaterials that attracted our attention was carbon nanotubes (CNTs), particularly single-walled CNTs (SWNTs), which was prompted mainly due to recent revelations about three vital traits that are required for in vivo therapeutic and diagnostic (theranostic) purposes. One, even though there have been mixed reports about the toxicity of CNTs, intravenous injection of adequately processed formulations in animal models has yielded encouraging results concerning their biocompatibility; ${ }^{7-13}$ and CNTs have demonstrated a high degree of internalization into a wide range of cells that could intracellularly traffic through different cellular barriers. ${ }^{14-16}$ Two, after favorable attachment to ligands, CNTs have shown a high degree of specificity to targeted cells and tissues. ${ }^{16-19}$ Three, the high NIR radiation absorption and thermal excitability of CNTs, affords minimally invasive photothermal (PT) and photoacoustic (PA) detection, as well as PT ablation of targeted tissue. ${ }^{2,16}$ This paved the way for integrated nanotechnology-based therapeutics and diagnostics, also popularly known as nanotheranostics, using CNTs and their hybrids (such as golden CNTs). 2,16,17,20

Despite recent progress, several challenges must be overcome before CNTs reach their full clinical potential. Particularly, issues like opsonization, phagocytosis by macrophages, and sequestration to the liver and spleen for eventual elimination from the body, should be addressed, which are major obstacles in the translation of NPs to clinical practice (Figure 1). This review focuses on strategies developed to coat and render CNTs transparent to nonspecific interactions with blood proteins, including components of the immune system, in order to improve blood circulation times and also to address biocompatibility and specific targeting issues, which are vital to the realization of their translation into clinical practice. While this review is not comprehensive, we discuss the importance of "stealth", meaning reduced uptake by the mononuclear phagocyte system, to improve the viability of CNTs as future theranostic agents, as well as the physicobiochemical properties that govern imparting of these traits. Also, we outline and analyze potential strategies that are currently being used to impart stealth characteristics to CNTs. Finally, we conclude with a discussion on the current needs and future directions of this research area.

\section{Challenges and opportunities Nanotubes as theranostic agents}

CNTs are hollow cylindrical tubes with high aspect ratios corresponding to $100-300 \mathrm{~nm}$ in length for processed CNTs and up to 2-5 $\mu \mathrm{m}$ for unprocessed CNTs, and diameters of 1-50 nm, depending on the types of CNTs. There are currently three known types of CNTs based on the number of rolled layers of graphene: SWNTs with a single layer (0.8-1.2 nm wide); double-walled CNTs (DWNTs) with two layers (1-2 nm wide); and multi-walled CNTs (MWNTs) with multiple concentric layers (5-50 nm wide). Ever since their discovery in $1991,{ }^{21}$ CNTs have attracted a lot of attention due to their unique structure and properties. They are known to be one of the most versatile nanomaterials around, as they exhibit superlative electronic, electrical, optical, thermal, magnetic, mechanical and, recently, biological properties. ${ }^{16,22}$ We will focus on the optical, thermal, and biological properties of CNTs since they are pertinent to the successful design and implementation of in vivo nanotheranostic agents. SWNTs exhibit band-gap fluorescence in the 800-1,600 nm wavelengths of the NIR spectrum. ${ }^{6}$ Photoexcitability of SWNTs in the NIR range is of extreme significance for biological applications, since living cells and tissues are virtually transparent to NIR radiation in the range of $750-1,200 \mathrm{~nm} .{ }^{16,23}$ Hence, the NIR responsiveness of SWNTs makes them ideal candidates for bioimaging and in vivo NIR laser targeting. Besides their ability to absorb and fluoresce NIR radiation, SWNTs act as perfect heat accumulators, where they confine the generated heat in its clusters, reducing heat loss to the surroundings. ${ }^{16,24}$ This critical property of SWNTs can eventually help in reducing extensive damage to the surrounding normal tissues by localizing the heat to small pockets around itself. ${ }^{16,20}$ SWNTs also have a high melting point of approximately $1,366 \mathrm{~K}$, which would ensure a negligible loss of structural integrity during the PT nanotheranostics. The combined optical and thermal properties have encouraged us to perform experiments on bacterial and mammalian cells in vitro, accompanied by PT and PA monitoring and PT damage using neodymiumdoped yttrium aluminum garnet (Nd:Yag) infrared laser pulses. 2,16,20,24-27 The results demonstrated the viable use of SWNTs as not only effective targeting agents, but also as high-affinity biomolecular interactive agents. 


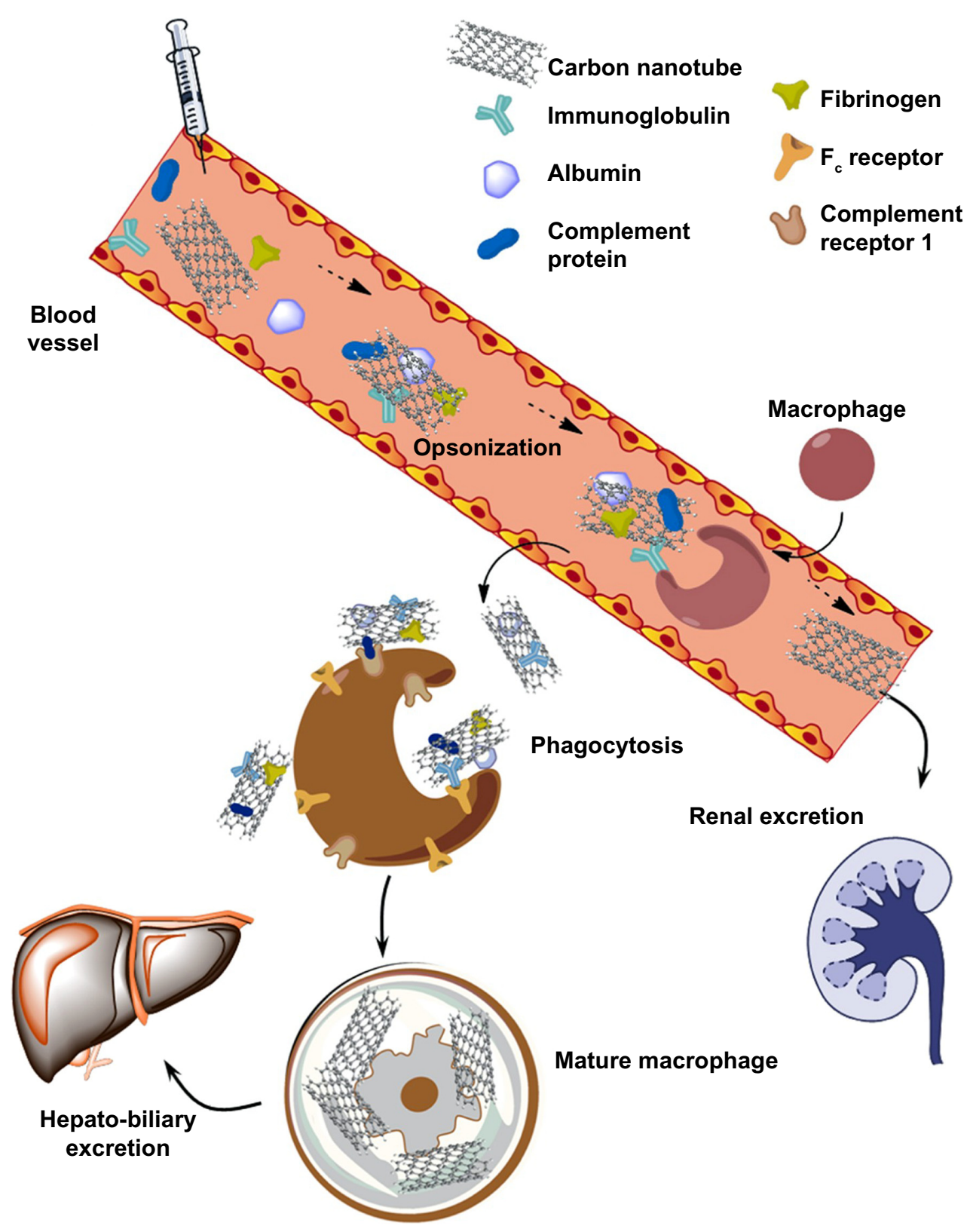

Figure I In vivo clearance pathway of CNTs after binding to opsonins and after subsequent recognition by macrophages in the blood vessels.

Notes: The macrophages engulf the CNTs and sequester them to the hepato-biliary organs, such as the liver and spleen, for excretion. Processed short CNTs with favorable dimension, orientation, charge, and functionalization are eliminated through the renal excretory system.

Abbreviation: CNTs, carbon nanotubes.

In addition, SWNTs possess the ability to passively transport themselves spontaneously through the cell membrane of animal cells, as was recently shown in some in vitro studies. ${ }^{14-16}$ This is dependent on the type, dimensions, and nature of the CNT construction. ${ }^{28,29}$ Henceforth, SWNTs have been demonstrated to act as transfection agents to transport deoxyribonucleic acid (DNA), ribonucleic acid, and other small biological moieties inside cells. ${ }^{17,30}$ The ability of SWNTs to interface with many of the biopolymers, as well as living cells on the whole, has prompted a wide array of biomedical experiments. ${ }^{17,19,20,24-27,31-34}$ The most important reason behind using CNTs as an in vivo vector is their shape and structure. The rod-shaped CNT with its high aspect ratio (ratio of length and diameter) provides an ample surface area for interacting with cell surfaces, while also affording favorable conformation to the attachment of ligands in multiple ways (Figure 2). For example, in oxidized SWNTs, their ends/tips with carboxyl groups $(\mathrm{COOH})$ can be used to attach certain ligands with favorable functional groups like amines, and the hydrophobic sidewalls can be 


\section{Non-covalent functionalization of CNT}

A

A Hydrophobic interaction

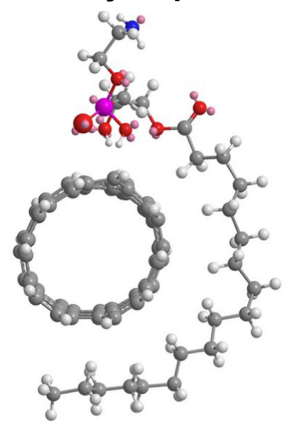

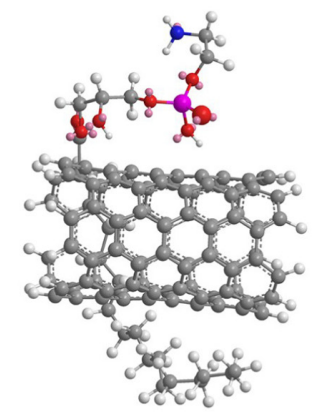

Pi-stacking interaction

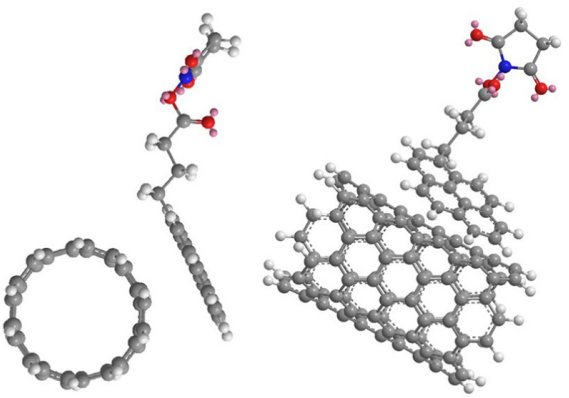

Covalent functionalization of CNT

B

1,3-dipolar cycloaddition
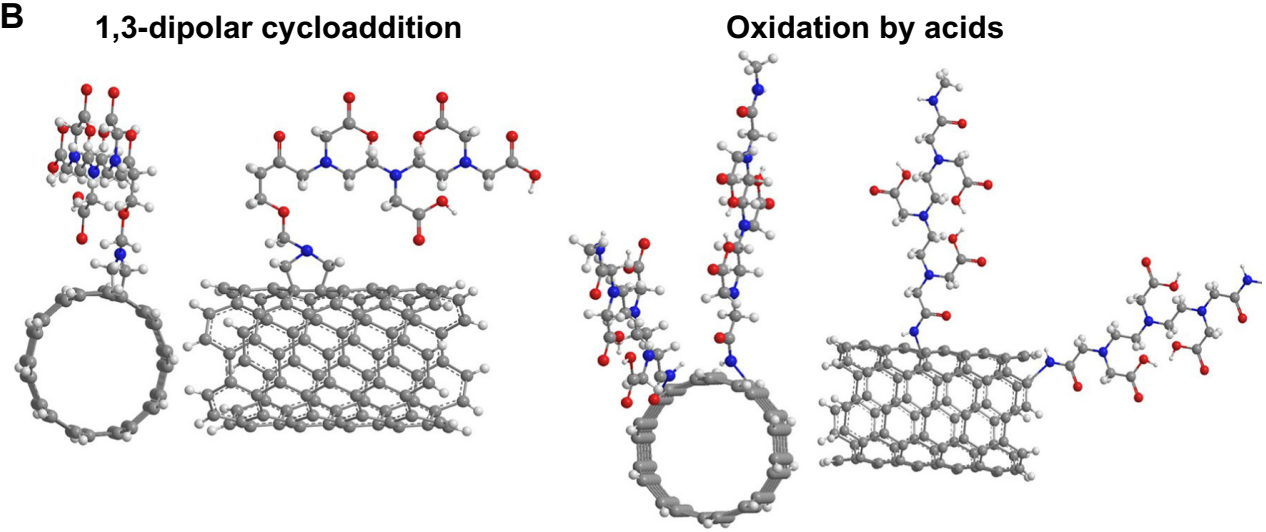

Figure 2 Modes of CNT functionalizations.

Notes: (A) Noncovalent functionalizations on the CNT surface are commonly achieved through hydrophobic and $\pi$-stacking interactions. (B) Covalent functionalizations are commonly performed using 1,3-dipolar cycloaddition and oxidation by acids.

Abbreviation: CNT, carbon nanotube.

used to attach ligands with continuous conjugated systems for $\pi-\pi$ interactions. This allows for multifunctionalities and for a myriad ways to design applications where such traits are highly required.

\section{Nanotube biofunctionalization}

The architecture and surface chemistry of CNT allows for ligands to be attached in four different ways: covalent bonding; noncovalent hydrophobic attraction; noncovalent $\pi-\pi$ stacking; and noncovalent electrostatic attraction (Figure 2). ${ }^{35}$ The covalent bonding of ligands to CNT is performed using the carboxyl functional groups that are introduced during the processing of pristine CNT, as a result of acid-induced oxidation. ${ }^{36,37}$ The $\mathrm{COOH}$ groups on the CNT's surface can be used to form amide linkages with ligands possessing amino groups. However, in some applications, this chemical coupling is not favored due to the purported effect of disrupting the electronic property of the CNT, and also due to the indeterminate number of $\mathrm{COOH}$ groups that can be introduced on the CNT, which is highly random and difficult to control. A given CNT can have $\mathrm{COOH}$ groups introduced on its entire surface, only partially, or on the tips, which could interfere with downstream control of ligand attachment, potentially leading to non-uniform distribution. For any particle that is chosen for an in vivo theranostic application, we believe it is imperative that the coating of ligand on its surface has to be complete and uniform, so that the actual surface of the particle that will come in contact with the surrounding fluids can be minimal and prevent nonspecific protein adsorption - otherwise known as a protein corona (Figure 3).

Prevention of protein corona formation is a prerequisite for efficient transportation, improved blood circulation halflife, and biodistribution of the particle. Noncovalent functionalization usually refers to coating the CNT with polymers and macromolecules such as polysaccharides, whole proteins, nucleic acids, and lipids including a combination of polymers and macromolecules as in 1,2-distearoyl-sn-glycero3-phosphoethanolamine-N-[amino(polyethylene glycol)] conjugates (Figure 4). This strategy preserves the structural 


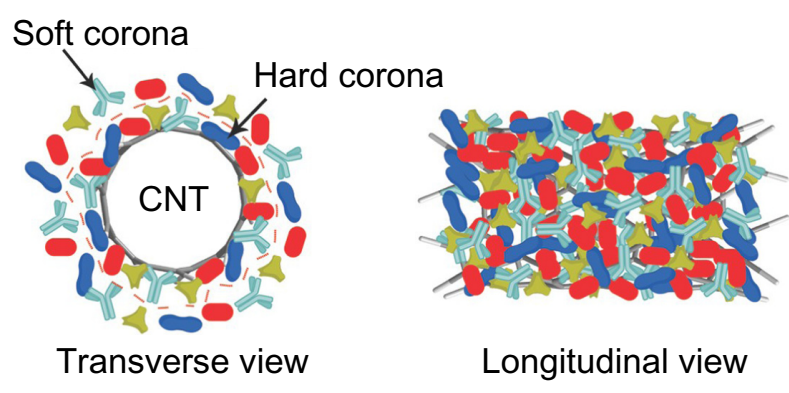

Figure 3 Protein corona formation on the CNT surface as a result of exposure to serum proteins.

Notes: A dense and tightly-bound layer known as the hard corona is directly formed on the CNT sidewall, and a loosely-bound layer known as the soft corona is formed on top of the hard corona.

Abbreviation: CNT, carbon nanotube.

and electronic integrity of the CNT by associating with the CNT surface indirectly through hydrophobic interactions. Noncovalent hydrophobic attraction is possible between the hydrophobic sidewalls of the CNT and hydrophobic domains located in the ligands..$^{25,38-41}$ For example, in proteins, there are many core hydrophobic domains, which unfold and interact with other hydrophobic materials. Polysaccharides, such as starch, have been shown to wrap on the CNT surface through hydrophobic interactions. ${ }^{40}$ The iodine molecule in the starch-iodine complex undergoes displacement by the CNT, thereby enabling the hydrophobic domains of starch to interact with the hydrophobic sidewall of the CNT. Another example of a hydrophobic interaction is the adsorption of phospholipid (PL)-polyethylene glycol (PEG) moieties to $\mathrm{CNT}^{42}$ The two hydrocarbon chains adsorb on the CNT sidewall, and the hydrophilic PEG groups render the CNT water soluble and biocompatible. However, noncovalent

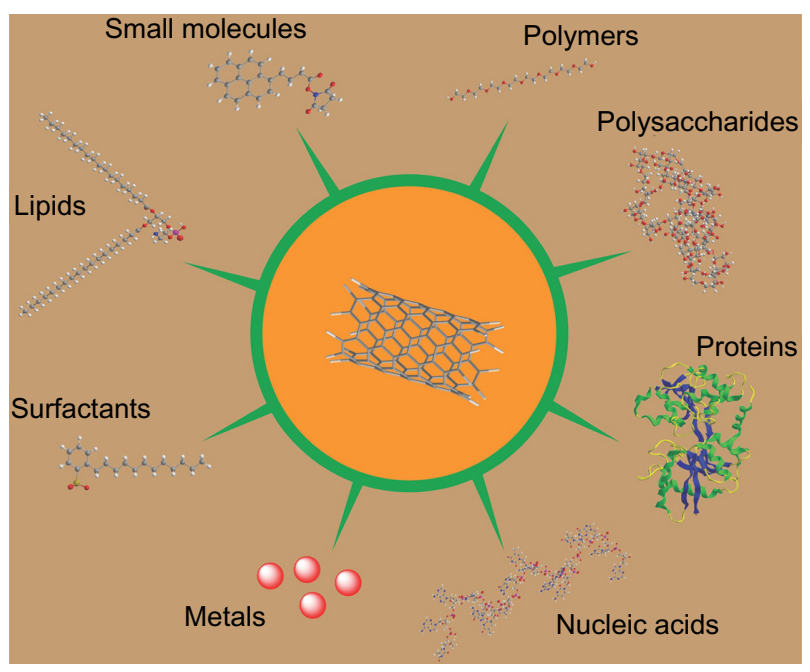

Figure 4 Various hybrid carbon nanotube conjugates that have shown promise for in vitro and in vivo systems. functionalization of CNT is prone to competitive adsorption and desorption in a rich medium, such as blood, due to the weak and transient nature of the interaction at physiological temperatures. This can easily negate the effect of the functional groups on CNT, as the "naked" CNT is now susceptible to tagging for elimination through nonspecific protein adsorption in the blood.

Aromatic compounds, due to their unique structure, possess $\pi$ electrons that complement the $\pi$ electron lattice of the CNT. This results in $\pi-\pi$ stacking of the aromatic rings on the CNT sidewall without directly coming in contact with the surface. ${ }^{43,44}$ It is CNT's unique electronic structure that makes such versatile bonding possible, owing to the presence of delocalized $\pi$ electrons. Pyrene is one such aromatic compound with the versatility to attach functional groups at one terminal for conjugation with various ligands. It has been demonstrated that proteins can be conjugated to CNT through an amino group reactive pyrene derivative. ${ }^{43}$ The same $\pi-\pi$ stacking mechanism can be used to helically wrap DNA on CNT, making them soluble in aqueous solutions, while also enabling separation of metallic CNT from semiconducting CNT. ${ }^{41}$ The helical isoalloxazine assembly of a low molecular weight (MW) compound, flavin mononucleotide, hydrogen bonds with the CNT chirality and helps in improving CNT dispersity and aqueous solubility. ${ }^{45}$ Due to the ease of use and control afforded by noncovalent modifications of CNT, we have extensively used $\pi-\pi$ stacking, as well as hydrophobic and electrostatic attraction to conjugate a wide variety of compounds, polymers, and biomolecules on the CNT sidewall. ${ }^{25,39,44}$ Another aspect of a noncovalent conjugation strategy is the versatility it imparts for downstream theranostic applications such as PT therapy and PA imaging. It has been shown that SWNT solubilized using a polymer carrying anthracene groups and irradiated with NIR light is able to dissociate the polymer from the surface of the SWNT and cause flocculation. ${ }^{46}$ Similarly, adsorbed species such as the chemotherapeutic drug, doxorubicin, can be desorbed from the SWNT for release into the surrounding medium using pulsed NIR light. ${ }^{47}$ Therefore, polymers and molecules that can be adsorbed on the CNT surface through weak bonds, such as through $\pi$ - $\pi$ stacking and hydrophobic interactions, provide an added advantage due to the weak and facile nature of the bond. Through this technique, highly precise and controlled drug release mechanisms can be achieved with far-reaching implications for nanomedicine. At the same time, caution should be applied in thoroughly evaluating these conjugates for their stability in various solvents and biological mediums before using them in biomedical studies. 
Processed CNTs (ie, CNTs oxidized using acids) possess numerous charged groups on their sidewalls, such as carboxyl and sulfonic acid, which can be used to electrostatically bind to other charged moieties. ${ }^{36}$ This is a conjugation scheme that requires not only a high degree of control over the compounds used, but also optimization of the buffer solution to prevent any cross-interactions with other charged groups in the medium, or that are later encountered in the interacting milieu.

It is hard to imagine that any other competing NPs have such a varied and suitable repertoire of properties for biomedical applications. Gold NPs, quantum dots, titanium oxide NPs, and so on, have their own pros and cons; however, it is CNTs' right mix of structure, shape, and optical properties, as well as their behavior in the biological environment that make them a frontrunner amongst contemporary NPs for suggested biomedical applications. Despite these advantages demonstrated by CNTs, they also possess certain inherent disadvantages that make them difficult materials to process and handle. CNTs have a high propensity to bundle up due to the van der Waals attractive forces existing between the individual tubes. ${ }^{48}$ This leads to complex entanglements that make them very difficult to disperse in solutions. The sp2 chemical bonding and architecture also make them hydrophobic, thereby resulting in extremely low solubility in aqueous solutions. It is common knowledge that all living systems are water-based and, therefore, aqueous solubility is a prerequisite for any potential biomedical applicability. Converting pristine CNTs, which are manufactured and supplied as a powder form, from an extremely long, bundled, and hydrophobic state into a shortened, individual, and hydrophilic state will be the first challenge that investigators usually face. Even though many strategies have been implemented to render CNTs as hydrophilic (such as treatment with surfactants Triton $^{\mathrm{TM}}$ X [Sigma-Aldrich, St Louis, MO, USA] and sodium dodecyl sulfate), there is a need for unique and novel solutions to make the CNTs not only water soluble, but also biocompatible. Surfactants are usually considered to be toxic to animal cells, as they denature the cell membrane and eventually lead to cell disruption. ${ }^{49}$

\section{Nanotube biocompatibility}

While it is important to assess the toxicity of pristine CNTs on vital organs and whole animals for understanding the potential risks associated with their usage, it is equally important to carefully assess the biocompatibility of processed and functionalized CNTs. This gains prominence given that well-dispersed and functionalized CNTs, which are currently used for biomedical applications, could be eventually used in translational studies. A wide array of cell types have been used to characterize the cytotoxicity of CNTs, and the data have been inconsistent and inconclusive. ${ }^{50-52}$ This is mainly due to the lack of standard and customized techniques available to assess CNT toxicity. ${ }^{53}$ For example, use of different cell types and diverse doses of CNTs for each study by different research groups has only led to a lack of consensus on this issue. It has been shown that viability studies of cells incubated with CNTs and assessed using the commonly used MTT assay (3-[4,5-dimethylthiazol-2-yl]-2,5-diphenyltetrazolium bromide) yielded false positives due to the physical attraction of SWNTs with MTT-formazan crystals, thereby interfering with the viability study. ${ }^{54}$ However, the use of its water-soluble counterpart, WST-1 (2-[4-iodophenyl]-3-[4nitrophenyl]-5-[2,4-disulfophenyl]-2H-tetrazolium) yielded a more plausible result as no SWNT-salt aggregations were observed. Interestingly, use of three-dimensional cell culture models have shown no cytotoxicity when both purified as well as oxidized SWNTs were used, when compared to twodimensional cell cultures. ${ }^{55}$ Since three-dimensional cultures closely represent the in vivo environment, it is assumed that the close cell-cell interactions lead to increased population viability and, at the same time, these adhesions prevent the uninhibited penetration of SWNTs. ${ }^{55}$ The role of the electronic structure of CNTs on biocompatibility has also been recently investigated. Metallic SWNTs typically have higher reactivity than semiconducting SWNTs ${ }^{56,57}$ Accordingly, it was observed that cytotoxicity of metallic SWNTs was higher on bacterial cultures when compared to semiconducting SWNTs. ${ }^{58}$ This is attributable to increased oxidative stress in bacteria caused by metallic SWNTs, due to increased glutathione activity. The role of the electronic structure of CNTs on the cytotoxicity of human cells also needs to be investigated, given that new and improved label-free, highresolution techniques have emerged that can selectively distinguish semiconducting SWNTs from metallic SWNTs with submicron accuracy in living systems. ${ }^{59}$ Besides these factors, various parameters, such as CNT surface properties and the presence/absence of impurities, also influence cytotoxicity studies to a great extent.

\section{Nanotube surface chemistry}

Investigators have reported extensively on the adverse effects of pristine CNTs on various organs, organ systems, and on the general well-being of experimental animals. ${ }^{60-63}$ In cellulo studies also suggested that the rope-like agglomerates of unprocessed SWNTs were found to be more cytotoxic than 
well-dispersed walled nanotubes on mesothelioma (MSTO$211 \mathrm{H})$ cells, even more than asbestos. ${ }^{52}$ The agglomerates, which are micron sized in diameter and length, are known to cause inflammatory reactions such as granulomas in the reticuloendothelial system (RES) organs such as the lungs, liver, and spleen through a process known as "frustrated phagocytosis". ${ }^{62}$ This occurs when activated macrophages unsuccessfully attempt to engulf the large agglomerates and proceed to form granulomas - a subtype of chronic inflammation. Therefore, the need for well-dispersed CNTs for biomedical applications cannot be more overemphasized. CNTs are capable of forming well-dispersed suspensions in aqueous solutions after oxidation in acid solutions, mainly due to the formation of carboxyl groups.$^{64}$ Even higher degrees of aqueous solubility can be attained by functionalizing the sidewalls with hydrophilic moieties, such as polymers. Studies on Chinese hamster ovary cells and Jurkat human T-lymphoma cells using glycopolymer-coated SWNTs and unfunctionalized SWNTs have demonstrated lower cytotoxicity for the functionalized SWNTs. ${ }^{65}$ Also, unfunctionalized SWNTs provoked the secretion of proinflammatory cytokines by macrophages in comparison to amino group-functionalized SWNTs. ${ }^{66}$ Some earlier studies using covalently conjugated streptavidin-SWNTs demonstrated extensive cell death of human promyelocytic leukemia HL60 cells after 2 days. ${ }^{67}$ In vivo studies using functionalized CNTs have shown good biocompatibility and faster clearance rates. The degree of chemical functionalization has proven to be the most important determinant of toxicity, with the higher degree of surface functional groups causing lesser accumulation in the liver and faster clearance through the renal system (Figure 5). In a study using 99mTc-labeled MWNTs, surface carboxyl functional groups with a density of $>3 \mu \mathrm{mol} / \mathrm{mg}$ and lengths $<500 \mathrm{~nm}$ of individualized nanotubes have demonstrated rapid clearance and low toxicity. ${ }^{8}$ Interestingly, the nature of the functional group, carboxyl or ammonium, was not critical to the low toxicity exhibited by the highly individualized CNT with multiple surface functional groups. ${ }^{13}$

\section{Nanotube impurities}

CNTs synthesized by both the arc-discharge process and chemical vapor deposition contain a high degree of catalytic impurities such as $\mathrm{Fe}, \mathrm{Co}$, and $\mathrm{Ni}^{64}{ }^{64}$ The cytotoxicity of these metals is well known and well documented. It was demonstrated that rat macrophages and human A549 lung cells, when exposed to unpurified CNTs, released reactive oxygen species intracellularly due to cell oxidative stress. ${ }^{68}$ Other cytotoxic effects include apoptosis; decreased adhesiveness and inhibition of proliferation of human embryo kidney cells; increased intracellular lipid hydroperoxides and the lowering of low MW thiols in murine macrophages; and lower cell proliferation rates in human epidermal keratinocytes. ${ }^{69-71}$ However, when highly purified CNTs were incubated with $3 \mathrm{~T} 3$ and HeLa cells, no cytotoxicity was observed. ${ }^{72}$ In another study, when A549 cells were separately treated with CNTs with $2 \%$ and $15 \%$ metal residues (predominantly $\mathrm{Fe}$ ), cells demonstrated reduced viability with $15 \%$ impurities in comparison to those treated with $2 \%$ impurities. ${ }^{73}$ The

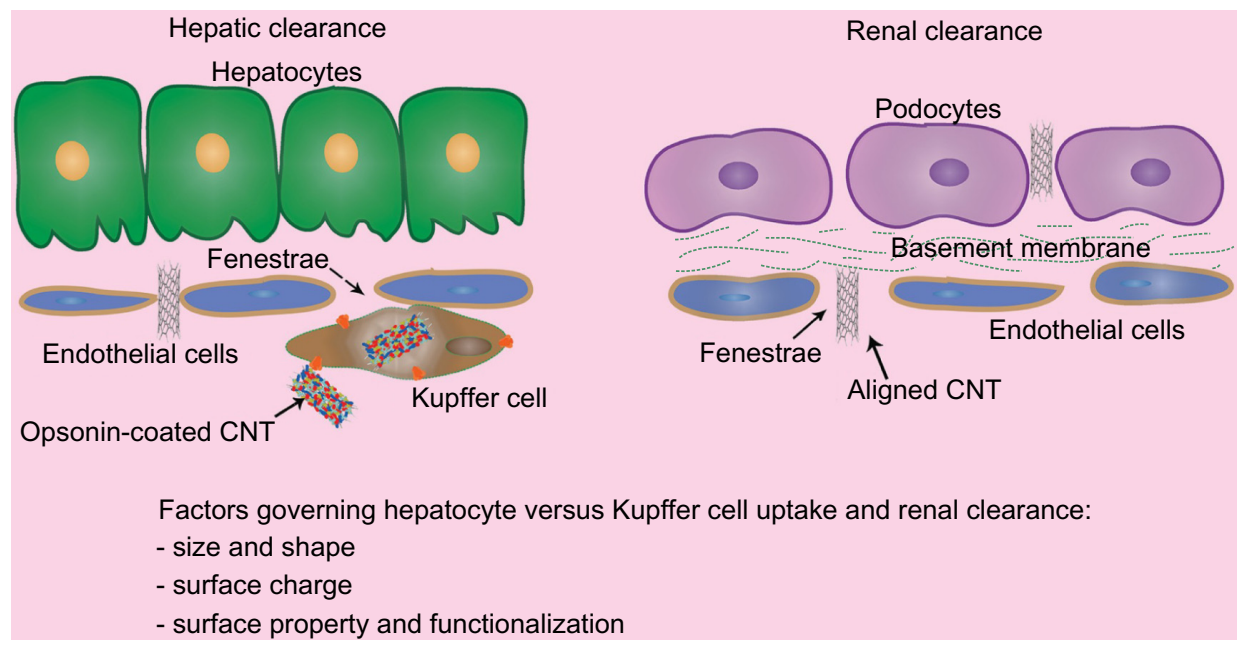

Figure 5 Hepatic and renal cellular ultrastructure illustrating the clearance mechanisms and physicochemical factors that contribute to effective clearance of CNTs. Notes: Those opsonin-coated CNTs that escaped being phagocytized by macrophages in the blood are eventually recognized and internalized by resident macrophages in the liver and Kupffer cells. Depending on certain physicochemical characteristics of CNTs, such as size and surface properties, some CNTs traverse the fenestrae in the endothelial layer of the liver sinusoids and enter the hepatocytes for eventual excretion through the hepato-biliary-fecal route. In the kidneys, however, only shortened CNTs with favorable charge characteristics and proper alignment can traverse the narrow (ie, $5 \mathrm{~nm}$ ) pores. Abbreviation: CNT, carbon nanotube. 
most commonly used method to remove the impurities involves acid treatment of CNTs to oxidize the catalysts. As a result of this procedure, carboxyl and hydroxyl groups are introduced on the CNT sidewalls. ${ }^{64}$ Surprisingly, it has been shown that purified CNTs are more cytotoxic when compared to unpurified CNTs, as demonstrated on human intestinal cells, Caco-2. ${ }^{50}$ This can be attributed to the carboxyl and hydroxyl groups on the CNT surface. It can, therefore, be reemphasized that rendering CNTs soluble in aqueous solutions does not guarantee the biocompatibility of the CNTs. Hence, it is imperative that further functionalization of the CNT surface is needed in order to shield the sidewall from the surrounding environment.

Interestingly, impurities such as Fe can be used advantageously for enabling the magnetic resonance imaging (MRI) of CNTs. Superparamagnetic iron NPs are known to influence the relaxation time of nuclear magnetization, and they have been used extensively as MRI contrast agents. This was tested using high-pressure carbon monoxide (HiPCO) SWNT with iron oxide NPs attached to one of the nanotube tips and wrapped with DNA to assist in dispersion. The SWNTs were internalized by macrophages and imaged in vitro using MRI. ${ }^{74}$ In another study, pristine SWNT with Fe impurities $(10 \% \mathrm{w} / \mathrm{w})$ was used for studying the in vivo biodistribution of systemically injected SWNTs using proton MRI imaging. A significantly higher gradient echo signal was obtained from the spleen and kidneys for pristine SWNT in comparison to purified SWNT. ${ }^{75}$ It is yet to be seen if toxicologically tolerable amounts of impurities in SWNTs, such as $2 \%$ Fe determined using in vitro assays, could permit MRI imaging. In a following study, it was found that purified SWNTs with $2 \%$ impurities and functionalized SWNTs with $0.7 \%$ impurities did not yield a statistically appreciable signals, thus confirming a threshold value of $10 \% \mathrm{Fe}$ impurities required for significant MRI contrast. ${ }^{76}$ Importantly, this study corroborates that $2 \% \mathrm{Fe}$, as impurities, can be tolerated in vivo, as no acute toxicological effect was observed in the liver using high-resolution magic angle spinning and quantitative real-time polymerase chain reaction analysis.

\section{Nanotube biodistribution and biokinetics}

Many pharmaceutical companies seek the development of novel drug delivery systems to deliver the correct dose at a specific site. The failure of traditional therapeutics in delivering precise and controlled amounts of a drug to targeted sites has often led to severe unwanted side effects, mainly as a result of administering excessively high doses. ${ }^{4}$ Novel nanomaterials such as CNTs are poised to address this issue due to the wide variety of targeting moieties that can be functionalized onto their surface. This new modality in therapeutics, through precision targeting, is expected to reduce systemic side effects and improve clinical outcomes. Usually, NP-based therapeutics are reliant on carefully selecting the right type of NP, wherein the NP simply serves as a vector to carry the therapeutic agent (such as antitumor drugs). To cite a few examples, the US Food and Drug Administration has approved some nanoparticulate and microscale drug delivery systems: Abraxane ${ }^{\circledR}$ (paclitaxel conjugated to albumin particles; Celgene Corporation, Summit, NJ, USA); AmBisome (amphotericin B liposome; Astellas Pharma US, Inc, Northbrook, IL, USA); Myocet (doxorubicin liposome; Enzon Pharmaceuticals, Inc, Picastaway, NJ, USA); Elestrin ${ }^{\circledR}$ (estradiol complexed to calcium phosphate NPs; Meda Pharmaceuticals Inc, Somerset, NJ, USA), and so on. ${ }^{77}$ The common theme in this list of nanoparticulate formulations is that almost all of them have the active component (the therapeutic agent) as the functionalized conjugate, and the carrier serves only for translocating it to the target site. However, the uniqueness of CNT stems from the fact that it can serve not only as a vector, but also as a theranostic agent due to its unique optical and thermal properties.

Generally, good therapeutic and clinical success can be achieved if four important parameters can be satisfied: low toxicity; increased blood residence time; high specificity; and excretability. From the moment the particle is injected intravenously until the moment it is completely excreted, it will have to defy the natural homeostasis (it is, understandably, an engineered "foreign" material in the body) and perform functions autonomously. One has to approach the selection and design of the NP with these factors in perspective, fully understanding that each phase is connected to the other and interdependent on each other's success. Surface functionalization of the CNT will aid in improving all four parameters (Figure 4). Besides surface functionalization, other parameters such as size and shape could also contribute to the "harmonization" of the CNT with the internal milieu. The good biodistribution, prolonged circulation time, blood hydrodynamics, and low clearance rate of CNT is an essential prerequisite for its proposed biomedical applications, such as tumor targeting and drug delivery. After intravenous injection, the CNT's blood residence time, organ biodistribution, and accumulation and clearance halflife are highly dependent on its surface characteristics and coating (Figure 5).

One of the critical events that occurs immediately after NP injection into the bloodstream is the formation 
of a biomolecular corona, also known as protein corona (Figure 3). This is the nonspecific interfacial reaction of blood proteins on the NP surface, which could play a decisive role in the biodistribution and biokinetics of NPs. ${ }^{78}$ Due to their high aspect ratio, CNTs can adsorb a large number of proteins on their sidewalls through: a $\pi$ - $\pi$ stacking interaction between the aromatic residues (for example, Tyr, Phe, and Trp) and the SWNT sidewall; electrostatic interactions; and hydrogen bonding. ${ }^{79,80}$ Proteomic analysis of the protein corona derived from human plasma adsorbed on the PEGylated SWNT revealed up to 500 proteins, and those derived from tissue culture media had up to 366 proteins adsorbed on the oxidized SWNT. ${ }^{80,81}$ However, it is important to delineate "hard" coronae (ie, a tightly bound nearmonolayer of biomolecules adjacent to the NP surface) from the "soft" coronae (ie, a more loosely associated and rapidly exchanging layer of proteins on top of the hard coronae) (Figure 3). ${ }^{78} \mathrm{~A}$ total of 181 proteins out of 366 constituted the hard corona in a study that employed cell culture media. ${ }^{80}$ The hard coronae represent not only the biomolecules that are the most abundant in the plasma and serum, which come in contact with the NP immediately upon introduction to the biological environment, but also quite possibly ones with the highest affinity to the NP surface. The affinity of the proteins to the NP surface is highly fleeting and varies depending on the type of surface coating, density of the coating, and charge. For example, it was determined that oxidized and charged CNTs (ie, with $\mathrm{COOH}$ groups) adsorb more proteins than pristine $\mathrm{CNT}$, and the most abundant proteins are titin, albumin, and apolipoproteins. ${ }^{80}$ Proteins, such as complement factors (C1q), even showed a preference for MWNT over SWNT and DWNT, mainly due to their larger dimension and unique geometry. ${ }^{82}$ Using HeLa cell lysates, it was shown that there is no strong correlation between protein affinity to the MWNT and their relative abundance in the medium, suggesting selectivity of the MWNT to certain proteins.$^{81}$ MWNTs behave as protein magnets by attracting and accumulating additional proteins, even after their surface is completely saturated by a protein corona ${ }^{82}$ This phenomenon can be attributed to strong protein-protein interactions. It is assumed that PEGylation of the NPs and other formulations prevents nonspecific protein adsorption in vivo. It was, however, demonstrated that not only does PEG not prevent protein corona formation, the pattern of adsorbed proteins also varies with the change in PEG conformation (Figure 6). ${ }^{83}$ When the PEG conformation transitioned from mushroom to mushroom-brush, human fibrinogen became more abundant than immunoglobulin (Ig)M and $\mathrm{IgG}$ on the

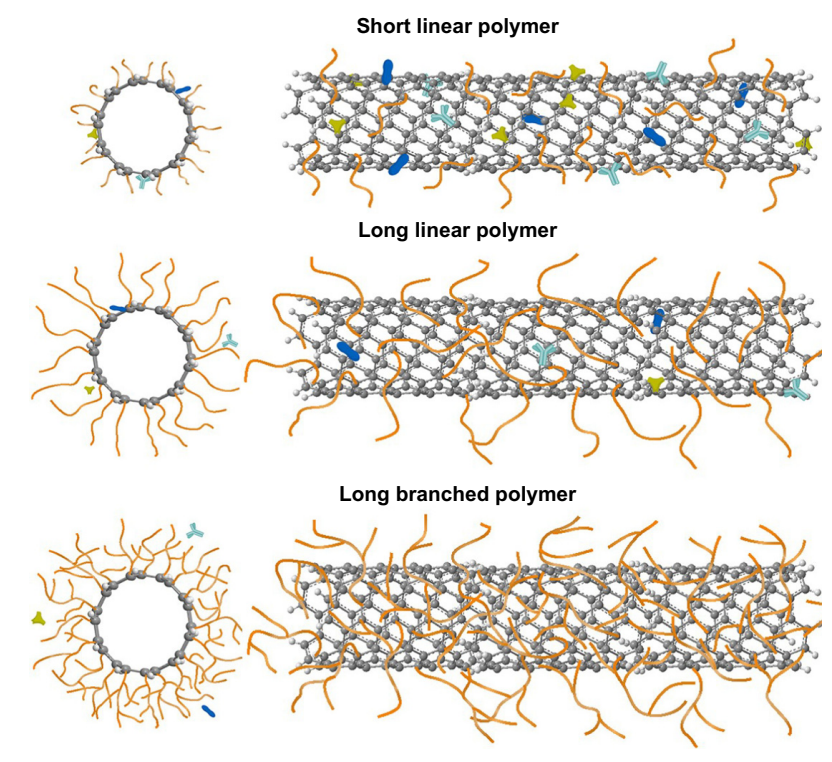

Figure 6 Shielding of the CNT surface using short linear, long linear, and long branched polymers and their effect on opsonization.

Notes: Long branched polymers are able to shield the CNT surface more effectively due to denser coverage on the CNT surface in comparison to short and long linear polymers.

Abbreviation: CNT, carbon nanotube.

SWNT surface. This further led to shorter blood circulation times, higher splenic versus liver accumulation, and faster renal clearance. It can be speculated that the quantitative differences in the proteins could alter the biodistribution profile. For example, higher IgM on SWNT could lead to preferential uptake by the liver. Most importantly, protein corona can also decrease the toxicity of CNTs by effectively shielding the surface from the external milieu. ${ }^{79}$ In addition, protein coronas might play a bigger role in CNT biokinetics in vivo, such as assisting in cellular interactions through biomolecular recognition, internalization, and signaling processes. ${ }^{84,85}$

The first biodistribution profiling of SWNT in mice was performed by Wang et al, ${ }^{86}$ where hydroxylated SWNTs were shown to be rapidly removed from circulation and distributed evenly throughout the body. The blood clearance half-life was 49 minutes ${ }^{86}$ Cherukuri et al ${ }^{87}$ reported that SWNTs dispersed using Pluronic ${ }^{\circledR}$ F108 (BASF SE, Ludwigshafen, Germany) surfactant had a blood circulation half-life of only 1 hour, as most of the SWNTs predominantly accumulated in the liver. ${ }^{87}$ Singh et a ${ }^{88}$ demonstrated SWNTs conjugated to a chelating agent, diethylenetriaminepentaacetic acid (DTPA), accumulated in kidneys (10.5\% injected dose [ID]), muscle (6\% ID), and skin (2\% ID) 30 minutes post-intravenous injection. ${ }^{88}$ The blood clearance rate was 3.5 hours. Almost similar blood clearance rates were observed by McDevitt et al ${ }^{89}$ on using 1,4,7,10-tetraa zacyclododecane-1,4,7,10-tetraacetic acid (DOTA), a more 
potent chelating agent compared to DTPA, conjugated to SWNT. ${ }^{89}$ The DOTA-SWNT conjugate accumulated in the kidneys (8.3\% ID), liver (17.8\% ID), spleen (14.3\% ID), and bone (2.26\% ID) 3 hours post-injection. PEGylation of the NPs has been extensively employed to render the xenobiotics resistant to opsonins, macrophages, and RES in general. Currently, it is the most popular agent to prevent the nonspecific adsorption of blood proteins in vivo. Liu et $\mathrm{al}^{42}$ reported branched PEG-PL with a MW of 2,000, 5,400, and 7,000 conjugated to SWNTs had varied blood circulation and biodistribution profiles. ${ }^{42}$ As the degree of branching increased from a MW of 2,000-7,000, there was an increase in the blood circulation half-life from 0.5-7 hours. Raman measurement on hepatic sequestration showed a decreasing trend: 70\% ID/g for 2,000 MW PEG-PL-SWNT and 30\% for 7,000 MW PEG-PL-SWNT at 24 hours post-injection. Therefore, higher degrees of branching of PEG leads to a more uniform and complete coating of the SWNT surface, which leads to a higher blood circulation half-life and delayed clearance (Figure 6). The blood residence time of PEG did not continue to demonstrate an upward trend beyond 7,000 MW PEG. This clearly demonstrates the need to sheath the CNT surface from the external environment by ensuring that most of (and if possible, the entire surface) is coated with the functionalizing agent. Also, there is a need for more coating materials that have PEG-like properties to prevent opsonization and RES sequestration. This will provide more options for investigators to coat various kinds of xenobiotics for in vivo applications.

The dynamics of the CNT in vasculature is essential to understand its interaction with the vessel wall, for both specific targeting to vascular targets, as well as for the prevention of nonspecific binding. The aspect ratio of the CNT is of particular concern, as there is speculation that it might damage the vasculature. This can be a difficult interaction to study in vivo. Recently, using synthetic microvascular networks, it was shown that polystyrene nanorods demonstrate sevenfold higher specific binding to vascular targets when labeled with targeting antibodies compared to unlabeled nanorods. ${ }^{90}$ Conversely, only a twofold advantage in specificity was observed with the nanospheres. Interestingly, nanorods demonstrated significantly lower nonspecific binding to the vessel wall when compared to nanospheres. However, the binding to vascular walls decreased as physiologically relevant hemodynamic shear stress increased. Even though CNTs were not used for this study, this has significant implications for all high aspect ratio NPs concerning the interplay with their shear dynamics and their interaction with vascular walls in vivo. In vitro studies have shown that in the event of nonspecific binding to vascular walls, the endothelial cells can transiently uptake SWNTs into the acidic vesicles, while not causing any cytotoxicity. Eventually, the cells release the nanotubes into the extracellular medium within 72 hours. ${ }^{91}$ The transient uptake and subsequent release of the nanotubes into the bloodstream bode well for CNT-based theranostics and, hopefully, allay any concerns regarding damage to vasculature due to the CNT's high aspect ratio. However, further in vivo studies using realtime high-resolution imaging techniques, such as intravital multiphoton microscopy, would help in better understanding this complex interplay. Recently, SWNTs have enabled high spatial resolution $(30 \mu \mathrm{m})$, deeper penetration $(1-3 \mathrm{~mm})$, and fast acquisition ( $<200 \mathrm{~ms})$ imaging of vasculature in the NIR 2 (1100-1400 nm) window that opens the door for further investigations into vascular hemodynamics and interactions with CNTs. ${ }^{92}$ When coupled to transient absorption microscopy, submicron resolution imaging can be achieved, which would permit investigating real-time nano-bio interactions with individual nanotube sensitivity. ${ }^{59}$

The liver and kidney are the two main organs involved in the clearance of xenobiotics, while pulmonary clearance cannot be ignored in the case of inhaled particles. In the liver, the parenchymal cells (hepatocytes) and phagocytic Kupffer cells represent the major pathway for clearance at the cellular level (Figure 5). Xenobiotics taken up by hepatocytes are eventually eliminated via bile into the feces, whereas those taken up by Kupffer cells are retained for a prolonged period of time and are enzymatically degraded in the phagolysosomal complex. Uptake by the hepatocytes versus Kupffer cells is governed by factors such as size of the NP, adsorbed opsonins on the NP surface, and charge of the NP. Typically, particles larger than $200 \mathrm{~nm}$ cannot cross the fenestrations of the liver sinusoids that drain into the parenchyma (Figure 5) $;{ }^{93}$ however, well individualized CNTs could traverse the fenestrae oriented along their long axis. It has been reported recently that shortened and well individualized CNTs with an average length of 200-300 nm pass through the glomerular membrane and get excreted through the renal system (Figure 5). ${ }^{94,95}$ This is partly because the glomerular capillaries in the kidney predominantly filter based on the size of the particles. Charge-based uptake was recently investigated using real-time intravital multiphoton microscopy, which revealed that negatively charged particles are taken up by Kupffer cells, and positively charged particles are taken up by hepatocytes. ${ }^{96}$ This was corroborated using carboxylated MWNT in a RAW 264.7 macrophage cell line that showed preferential uptake for oxidized MWNTs over PEGylated 
MWNTs. ${ }^{97}$ Protein corona formation inadvertently affects specific binding of the NPs to the numerous receptors present on the surface of the Kupffer cells (Figure 5). Since protein corona is an inevitable outcome of systemic injection of the NPs, it is highly likely that majority of the CNTs localize in the Kupffer cells. ${ }^{78}$

Until a few years back, it was assumed CNTs are nonbiodegradable and would accumulate and biopersist in organs, leading to chronic inflammation and eventually long-term toxicity. ${ }^{61,62}$ Recent evidence points to the possibility of addressing this issue through enzymatic degradation of the CNT in biological systems, thereby assisting in its elimination (Figure 7). Phagocytic cells such as macrophages and, notably, neutrophils have an abundance of myeloperoxidase (MPO), a peroxidase enzyme that generates hypochlorous acid and other reactive intermediates that are known to have enough oxidizing potential to break $\mathrm{C}-\mathrm{C}$ and $\mathrm{C}-\mathrm{H}$ bonds (Figure 7). ${ }^{98}$ Molecular modeling studies have revealed the binding of SWNT to the catalytic sites of the enzyme, which initiates the hypochlorite-mediated biodegradation of SWNT, assisted by other reactive intermediaries. ${ }^{99}$ Other peroxidases such as horseradish peroxidase and heme-dependent peroxidase are also capable of effectively degrading CNT. ${ }^{100,101}$ However, the effectiveness of degradation has a strong correlation to the abundance of the enzyme in respective cell types. For example, MPO-rich neutrophils degraded $100 \%$ of the SWNTs after 12 hours through the synergistic action of MPO and nicotinamide adenine dinucleotide phosphate oxidase, whereas macrophages with less abundant MPO only degraded $13 \%$ of the SWNTs at the end of 12 hours, which progressed to a more pronounced $50 \%$ after 48 hours of incubation. ${ }^{99}$ An in vivo study on MPO-deficient mice revealed that as a result of lower oxidation, the inflammatory response to SWNTs was higher than in the wild-type mouse, which led to a lower clearance rate. ${ }^{102}$ Other phagocytic cells, such as brain microglia, have also demonstrated partial biodegradation of MWNTs after 14 days of in vivo stereotactic administration into the mouse motor cortex. ${ }^{103}$ The partially degraded CNT could yet be a cause for concern not only due to its long-term proinflammatory potential, but also because of the molecular byproducts of the degradation pathway, such as polyaromatic hydrocarbons. However, upon administration of a partially degraded SWNT sample into the mouse pulmonary system, there were no signs of inflammatory markers in the pharyngeal aspirate. ${ }^{99}$ In contrast, nondegraded SWNTs showed acute inflammatory response accompanied by granuloma formation in the lungs. The biodegradation of CNTs, while encouraging, should be cautiously and thoroughly evaluated in various cell types, including the human microbiome, and the effect of the oxidative bimolecular machinery on different types of CNTs should be carefully assessed.

In environmentally relevant conditions, CNTs can be biodegraded by the cooperative activity of several microorganisms, as revealed by a study using ${ }^{14} \mathrm{C}$-labeled MWNT. ${ }^{104}$ Up to $6.8 \%$ of MWNT was transformed to ${ }^{14} \mathrm{CO}_{2}$ over a period of 7 days with the help of an external carbon source for cometabolism. However, $\mathrm{CO}_{2}$ was not the only byproduct of the degradation pathway, as other intermediaries such as 2-naphthol, 2-methoxy naphthalene, isophthalic acid, and so on, were also generated. Importantly, it was demonstrated that no single microorganism could efficiently degrade the

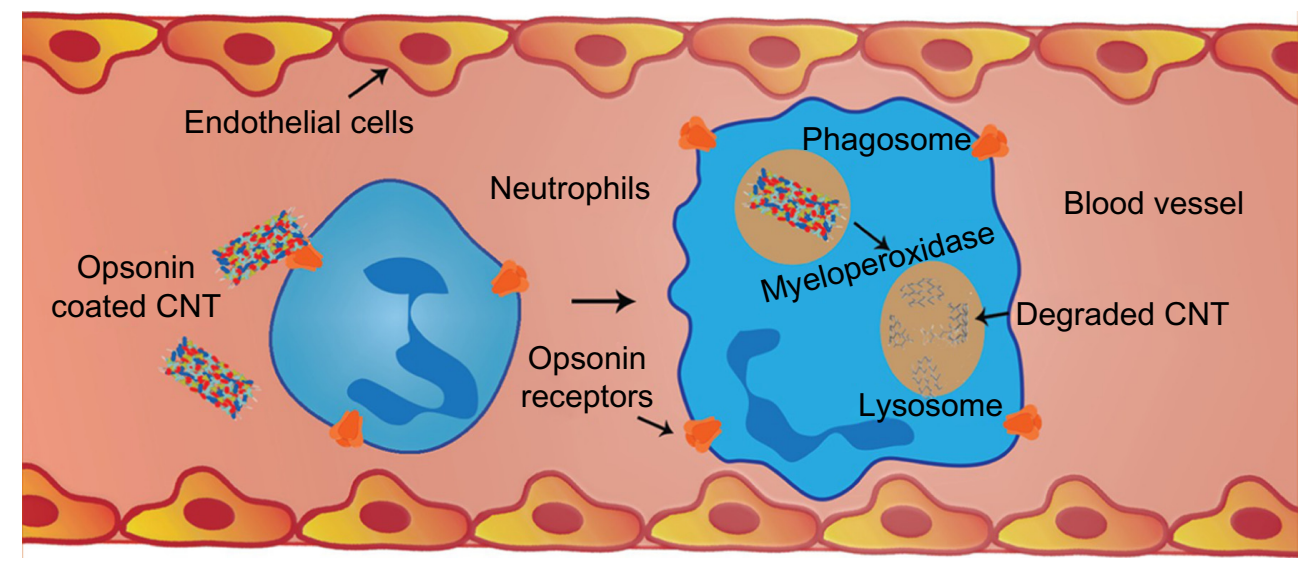

Figure 7 CNT biodegradation in vivo.

Notes: Circulating neutrophils expressing opsonin-binding receptors, such as Fc and complement receptors, in the blood are capable of internalizing and degrading opsonincoated CNTs. Myeloperoxidase, a peroxidase enzyme abundantly expressed in neutrophils, is capable of degrading the lattice structure of CNTs by breaking the C-C and $\mathrm{C}-\mathrm{H}$ bonds.

Abbreviation: CNT, carbon nanotube. 
MWNT. When a combination of different microorganisms was used, there was a tenfold increase in the biodegradation of MWNTs. This has important implications for the environment in general, as the environmental persistence of CNTs can be attenuated. In addition, it holds important implications for in vivo applications where the human microbiome could play a potential role in assisting further biodegradation of CNTs in the gastrointestinal tract and other sites with a sizeable population of microbiota. Degradation is also dependent on the type and surface functionalization of the CNT. An in vitro study has shown that SWNTs are oxidized and degraded more efficiently when compared to MWNTs. ${ }^{105}$ In addition, SWNTs with surface carboxyl groups are degraded efficiently when compared to pristine, ozone-treated and aryl-sulfonated SWNTs. ${ }^{106}$ A greater degree of carboxyl functionalization, which considerably improves the hydrophilicity of the CNTs, leads to an improved rate of degradation. ${ }^{107}$ Therefore, surface rendering of the CNTs and NPs in general for rapid and efficient elimination is an ongoing critical investigation that will eventually determine their potential for theranostics and, ultimately, clinical translatability.

\section{Need for stealth}

The immune system is a defense mechanism of the human body that acts against invading pathogens and foreign materials, and works on the basic "self/non-self" discriminatory rule. Anything considered harmful, including cells of the human body, are quickly eliminated. Macrophages are decorated with a wide arsenal of receptors hooked to the plasma membrane: 1) receptors for complement factor $\mathrm{C} 3 \mathrm{~b}$; 2) receptors for the Fc portion of immunoglobulins; and 3 ) receptors to mannose/fucose and other bacterial surface polysaccharides (Figure 1). ${ }^{108-111}$ Once the pathogen/foreign material that has already been tagged by opsonins comes in contact with macrophages, it is rapidly recognized by the receptors, which then initiate the phagocytic process where the particle is engulfed by the macrophage for intracellular digestion. Any undigested particles end up in the liver and spleen and are eventually excreted. ${ }^{112}$ It can then be safely agreed that CNTs would suffer the same fate as any pathogen entering the bloodstream (Figure 1). Opsonins, C3b, and IgG, which are present in the blood in substantial quantities, will readily attach to the hydrophobic sidewall of the CNT, which will tag them for macrophage recognition. Even in reports where whole-body biodistribution studies are conducted, ${ }^{42,89}$ after amenable functionalization with ligands that prolong blood residence times, there are high percentages of NPs that end up in the liver and spleen because materials injected in bulk, in order to improve bioavailability, often aggregate and precipitate. In such studies, the only way to effectively monitor the location of the particles is to have a high enough concentration to be able to trace them. Therefore, a probable solution to improve the blood residence time is to devise novel ways to coat the CNT with biocompatible ligands, which prevents the attachment of opsonins to the CNT by acting as a shield, and individually assess their performance in vitro and in vivo. By simulating the microenvironment of the blood vessel where such events are likely to take place, in a matter of 1-4 hours after injection, one can determine the behavior and fate of CNT and devise appropriate modifications to shielding if necessary. Shielding is a crucial step in rendering the CNT transparent to opsonins and deceiving the innate immune response to make believe it is not foreign. The success of any diagnostic or therapeutic precision targeting system cannot be taken for granted by overlooking the stealth mechanics of the given particle. Acquaintance with the factors and principles that normally decide the stealth behavior of a particle is required before we delve into the strategies used to coat CNTs.

\section{Physicobiochemical characteristics governing stealth nanotube agents}

\section{Size matters}

Physiological and anatomical parameters, such as blood transport, tissue diffusion, hepatic filtration, and renal excretion, along with the size of fenestrae in the end capillaries, dictate that the size of the particles is an important factor that ensures prolonged circulation times and high therapeutic efficacy for NPs. ${ }^{113}$ It was shown that in polystyrene particles, ranging from 50-500 nm, larger particles demonstrated higher levels of hepatic sequestration, thereby lowering their circulation times. ${ }^{114}$ Since the emphasis is on how NPs interact with opsonins and macrophages, the radius of curvature, which is related to size, will impact protein attachment and subsequent macrophage interaction. It has recently been shown that the NP size is not as important a determining factor for phagocytosis as it was thought to be. ${ }^{115,116}$ Instead, it was found that macrophages recognize NPs as a function of the number of opsonins that are attached, rather than the function of size. ${ }^{116} \mathrm{~A}$ recent study comparing SWNTs and titanium dioxide $\left(\mathrm{TiO}_{2}\right)$ NPs revealed that SWNTs adsorbed fewer plasma proteins, even though they had a larger surface area. ${ }^{117}$ SWNTs with a diameter of $0.8-1.2 \mathrm{~nm}$ are narrower than the smallest $\mathrm{TiO}_{2} \mathrm{NPs}(\sim 2 \mathrm{~nm})$, thus having a high radius of curvature, which tends to offer fewer attachment sites for plasma proteins than larger particles with a lower radius of 
curvature. Fewer opsonins on narrower particles such as SWNTs mean lower uptake by macrophages, which would translate to higher SWNT blood circulation times.

Some authors have demonstrated that for particles less than $100 \mathrm{~nm}$, there is minimal to no uptake by the macrophages, whether they are coated or not by opsonins. ${ }^{118,119}$ Therefore, it can be concluded that for particles with a high radius of curvature and a small size $(<100 \mathrm{~nm})$, the outcome is more promising when compared to larger particles with a low radius of curvature. This augurs well for SWNTs, which have an effective diameter of $0.8-1.2 \mathrm{~nm}$ and lengths that vary from $50-150 \mathrm{~nm}$ after processing. With such a narrow diameter and high radius of curvature, it would seem that SWNTs are ideally suited to escape opsonin binding, without any extraneous functionalization and coating. However, the length of the SWNT has a propensity to show a lot of variation, mainly due to the random nature of the shortening process employed. In vivo, shorter CNTs have shown propensity towards improved biodistribution and effective clearance through the kidney and liver. ${ }^{42,94,96}$ Shorter CNTs $(<200 \mathrm{~nm})$ are able to traverse the endothelial cell barrier through their fenestrae along both axes, which would potentially permit uptake by hepatocytes and excretion through feces. However, in kidneys, the physiologic pore diameter is significantly smaller (around $5 \mathrm{~nm}$ ), therefore permitting passage of only aligned SWNT, tip first and along the longitudinal axis (Figure 5).

\section{Effect of shape}

It has been demonstrated that the shape of NPs plays a greater role than their size in the process of phagocytosis by macrophages. Different shapes of polystyrene particles were synthesized and exposed to macrophages after opsonization with IgG. ${ }^{115}$ The $\Omega$-angle was defined as the angle formed between the point of contact of the cell membrane and the particle. Phagocytosis was not initiated at $\Omega>45^{\circ}$ for nonspherical particles. Opsonized and nonopsonized particles had the same $\Omega$ dependence. ${ }^{115}$ This essentially means that particle internalization is primarily dictated by the shape of the surface at the point of initial contact. For example, in a rod-shaped particle, internalization is initiated only if the tip/end of the rod comes in contact with the cell membrane of the macrophage, whereas no internalization happens if the point of contact is the sidewall. In a recent study, ${ }^{120}$ it was demonstrated that MWNTs undergo tip-first recognition and entry into cells. Through receptor clustering at the rounded tube tips, the cells initiate the uptake of the one-dimensional particles. Interestingly, this was observed only in tubes that had closed end caps, and not in open-ended tubes. Therefore, orientation of the rod prior to interacting with macrophage will play a great role in determining if internalization proceeds or not. Spherical particles are not faced with this complication and, therefore, will more readily undergo internalization. The shape of the SWNT clearly affords it an advantage in comparison to spherical and ovoid particles as extraneous and additional factors, such as orientation, do not play any role in their internalization. However, the orientation of SWNTs is crucial for in vivo clearance, particularly for shortened SWNTs, when in the glomerulus, as the small functional pore size of the endothelium-basement membrane-podocyte complex will only permit passage if the orientation is along the long axis (Figure 5). ${ }^{94,95}$

\section{Surface hydrophilicity}

Hydrophilicity, when compared to hydrophobicity, is known to greatly prevent nonspecific adsorption. ${ }^{121}$ Opsonins, IgG and complement factors, and plasma proteins in general adsorb more readily on hydrophobic surfaces than on hydrophilic surfaces (Figure 3). ${ }^{122-124}$ In vitro and in vivo studies have also demonstrated higher degrees of phagocytosis and increased sequestration to hepatic tissue of hydrophobic particles in comparison to hydrophilic particles. ${ }^{123,125-127}$ In order to counteract the attractive hydrophobic forces between the hydrophobic particle and proteins, polymers such as PEG are conjugated to hydrophobic surfaces. ${ }^{128}$ PEG, along with other similar polymers, are hydrophilic, inert (no net charge), biocompatible, and elicit no response from plasma proteins. ${ }^{128}$ The presence of the polymer coating on hydrophobic surfaces creates a water cloud, which serves as a potential barrier against plasma proteins by preventing any binding from occurring, while denying any attachment sites. ${ }^{129}$ The water cloud is formed due to the high affinity and linking of two to three water molecules by each polymer chain. ${ }^{130}$ The low attractive forces between the surface and proteins are due to the creation of minimum interfacial free energy by the water cloud. ${ }^{129}$ Besides polymers such as PEG, polysaccharides are also used, mainly due to their biodegradability. ${ }^{131}$ Pulmonary surfactants, such as CUROSURF ${ }^{\circledR}$ (Chiesi Farmaceutici, SpA, Parma, Italy) are able to nonspecifically bind to CNT and, in turn, be able to alter the composition of the protein corona as a result of precoating and altered hydrophobic interactions. ${ }^{132}$ It is believed that the protein corona decides the fate of the NP in vivo, and since the composition of the protein corona is dependent on the hydrophilicity of CNTs, the eventual fate can be controlled with adequate processing of CNTs. Even though precoating with polymers and surfactants, and 
rendering the CNT surface as hydrophilic, cannot completely abolish protein corona formation, it has the propensity to decrease and alter the protein profile that could enhance CNT's in vivo biodistribution and biokinetics.

\section{Surface property and charge}

By comparing NPs such as SWNTs, MWNTs, TiO ${ }_{2}, \mathrm{ZnO}$, $\mathrm{SiO}_{2}$, and quartz sand, Sund et $\mathrm{al}^{117}$ identified that adsorbed plasma proteins, such as fibrinogen, complement proteins, Igs, and apolipoproteins, did not vary, and they covered NPs irrespective of their surface properties. However, the binding efficiency of these NPs to cell lysate proteins varied based on surface characteristics. This is attributable to plasma proteins having glycosylated residues not commonly encountered among cytoplasmic proteins. The formation of protein coronas around NPs might also likely be due to alterations in their surface properties due to the $\mathrm{pH}$ of the surrounding environment. $\mathrm{TiO}_{2}$ interacts with proteins more effectively at pH 6 compared to neutral and near-basic $\mathrm{pH}$ values.

Most functional groups have a net charge and, even though they can independently contribute to the interaction dynamics with plasma proteins and macrophages, we will analyze them together in this section. Recent investigations using nanoshells have shown that positively charged particles have a higher rate of uptake by phagocytic cells in comparison to negatively charged and neutral particles. ${ }^{133}$ In another study, negatively charged PEG-single-walled carbon nanohorns displayed lower macrophage uptake. ${ }^{134}$ It is important to note that the density of the surface coating is equally important in determining cellular uptake. Different NPs can demonstrate highly discordant behavior when internalization is purely analyzed from a charge-based perspective. For example, recently, in vivo intravital real-time multiphoton microscopy of the liver revealed that positively charged mesoporous silica NPs was primarily taken up by hepatocytes, and negatively charged particles were taken up by phagocytic Kupffer cells (Figure 5). ${ }^{96}$ Likewise, a study using polystyrene NPs showed that negatively charged particles had a higher internalization rate into macrophages, and positively charged particles into monocytes. ${ }^{135}$ Highly charged surfaces, in comparison to neutral particles, attract complement factors. However, Liu et al $^{136}$ demonstrated that complement factors are not activated in the presence of neutral and negatively charged particles. Patel et al, ${ }^{110}$ on the other hand, demonstrated that positively charged particles became negatively charged in serum after the opsonization process. Experiments on polystyrene particles functionalized with amine, hydroxyl, carboxyl, and sulfate groups have demonstrated that amine groups, along with hydroxyl groups, activate complement proteins C3 and C4. ${ }^{13}$ Also, experiments on thiolated NPs have revealed shorter plasma half-lives, up to 3 hours, and preferential uptake by spleen. ${ }^{137}$ Therefore, the design of "stealth" NPs should incorporate several parameters that are important in collectively rendering the NPs transparent to the phagocytic cells of the RES.

\section{Strategies to shield CNTs}

Many strategies that are used to coat CNTs for efficient in vivo functionality have erupted over the last decade (Figure 4). While some studies have focused on the interaction of these hybrid CNTs with cells such as macrophages, lung epithelia, skin fibroblasts, and varied tumor cells at the cellular level, others have looked at their pharmacokinetics and biodistribution profiles in vivo. ${ }^{62,70,138-146}$ However, a majority of these strategies are focused at aiding the internalization of CNTs into their respective cell types for imaging and therapy. While this addresses the targeting component of the CNT-based nanoagents, it avoids the issue of opsonization when the particles are introduced into biofluids. It is possible that some of these novel coatings could impart their requisite "stealth" character to the CNTs. This, however, is seldom practiced due to a variety of considerations. A holistic approach, whereby the manufactured hybrid CNT nanoagents are tested and validated both for targeting and long circulation times in vivo, will lead to better assessment and confidence in their potential.

The strategies used to shield the CNTs can be broadly divided into noncovalent and covalent (Figure 2). The noncovalent modality for shielding CNTs have been demonstrated in order to improve blood circulation time and biodistribution. ${ }^{42,147}$ A hybrid construct made of lipid and PEG was used, where the lipid moiety interfaces with the CNT sidewall through hydrophobic interaction, and PEG affords improved biodistribution. The report suggests a stable interaction between the lipid moiety and the CNT surface throughout the study. This is important since dissociation of the lipid-PEG construct from the CNT will enable the ready adsorption of opsonins, along with various plasma proteins, for recognition and elimination by the RES pathway. However, in a recent study, ${ }^{148}$ it was shown that MWNTs pretreated with CUROSURF ${ }^{\circledR}$, a pulmonary surfactant consisting primarily of PLs, and incubated with blood plasma, underwent dynamic changes in the lipid coatings. The primary blood plasma lipids, such as cholesterol and triglycerides, appeared to bind to the MWNTs, and there was also a low association of phosphatidylserine (PS), one of the PLs from CUROSURF ${ }^{\circledR}$. 
In a related study, ${ }^{138}$ it was demonstrated that SWNTs coated with PS were recognized by professional macrophages and internalized through the endocytotic pathway. However, there was no recognition or uptake of phosphatidylcholine-coated SWNTs by macrophages. In apoptotic cells, PS is no longer restricted to the cytoplasmic side of the membrane and is exposed on the surface to the extracellular medium, thus enabling recognition by PS-specific receptors present on macrophages. Taking cues from such natural processes, one can envisage the development of artificial agents that exploit this machinery for effective in vivo roles. Can PLs devoid of PS be used as a potential "stealth" imparting moiety, provided their stability in an in vivo dynamic environment is reliable? Even though this concept is significantly compelling, it is early to conclude that MWNTs pretreated with lipids can be displaced by plasma lipids, and possibly even proteins, upon exposure to blood. This phenomenon is reminiscent of the Vroman effect, which originally described complex protein adsorption-desorption effects occurring on particle surfaces. ${ }^{149}$ Highly mobile proteins such as bovine serum albumin adsorbed on biopolymer surfaces were observed to be displaced by less mobile, but high-affinity, proteins such as fibrinogen. Besides the binding affinity and stability of the lipophilic interfacing moiety, it is also important to consider the hydrophilic interfacing group (PEG) that affords immunity to the CNTs against opsonins. For this, considering the high aspect ratio of CNTs and other rod-shaped NPs in general, length, density, and the conformational state of the polymer moiety plays a significant role in determining the stealth behavior of CNTs.

Liu et $\mathrm{al}^{42}$ demonstrated that increasing the length of the linear PEG chain from $2 \mathrm{kDa}$ to $5 \mathrm{kDa}$ increased the blood residence time from $\sim 1.2$ hours to 5 hours. However, there was negligible improvement in the blood residence time when $7 \mathrm{kDa}$ and $12 \mathrm{kDa}$ PEG were used. When branched $7 \mathrm{kDa}$ PEG chains were used, a dramatic increase in the blood residence time of $\sim 15$ hours was observed. ${ }^{150}$ Further studies carried out by Liu et al ${ }^{143}$ using different densities, ranging from $5 \%, 10 \%, 25 \%, 50 \%$, and $100 \%$ with both $2 \mathrm{kDa}$ and $5 \mathrm{kDa}$ PEG chains, brought about increasing blood circulation times, up to $\sim 12.8$ hours for $10 \%-5 \mathrm{kDa}$ PEG to $\sim 21$ hours for $100 \%-5 \mathrm{kDa}$ PEG. This suggests the effect of increasing linearity and density of polymer coating on the ability of hybrid CNT constructs to evade opsonins, thereby improving blood circulation times (Figure 6). Improved density translates to lowering the unoccupied gaps on the CNT surface that could potentially serve as attachment sites for opsonins. The biodistribution profile of the respective PEG chains demonstrate predominant uptake by RES organs such as the liver, spleen, and bone marrow, more so for the linear PEG chains. Long-term fates of the linear PEG-SWNT constructs in the animals showed that the majority of them were excreted via the biliary pathway into the intestine, and eventually through feces. The authors also alluded to renal excretion among a small percentage of SWNTs that were below the glomerular excretion threshold $(<50 \mathrm{~nm})$ at 24 hours post-injection. Interestingly, there was significant accumulation of the long circulating constructs with half-lives $>16$ hours, $50 \%-5 \mathrm{kDa}$ PEG and $100 \%-5 \mathrm{kDa}$ PEG in the skin ( $29 \%$ ID/g), which is comparable to tumor uptake $(\sim 23 \% \mathrm{ID} / \mathrm{g})$. In contrast, there was relatively lower uptake of $10 \%-5 \mathrm{kDa}$ PEG with blood circulation times of $\sim 12.8$ hours in the skin $(\sim 3 \%$ ID/g), as well as acceptable tumor uptake $(\sim 15 \% \mathrm{ID} / \mathrm{g})$. Accumulation of ultra-long circulating PEG-SWNT in skin is intriguing and presents unusual challenges that could potentially interfere with the purported applications such as PT and PA imaging and therapy, where there is a high probability of interference from the accumulated dermal SWNT lying in the beam path. Therefore, it seems that a good balance of optimal circulation times, tumor uptake, and low accumulation in the RES organs and skin by controlling the length and density of the polymer coating, will help in achieving a desirable outcome. However, these studies, have seemingly omitted evaluation of the biphasic pattern in plasma concentration profile of the hybrid adducts. Any injectable is eliminated from the blood through two phases: 1 ) an initial $\alpha$-phase, where the injectable is rapidly distributed to the tissues from the blood, followed by a pseudo-equilibrium phase where the drug equilibrates between the blood and tissue compartments; and 2) a gradual $\beta$-phase, where the drug is slowly metabolized and excreted. This would be helpful in determining the relationship between the different phases and organ-specific uptake - tumor uptake in particular.

The pan European groups of Bianco et $\mathrm{al}^{14}$ and Kostarelos et $\mathrm{al}^{151}$ have spearheaded the biological characterization and pharmacokinetics of covalently functionalized CNTs (Figure 2). Ammonium-functionalized CNTs were generated, to which DTPA, a chelating molecule, was linked to enable the complexation of $\gamma$-emitting radiometals such as ${ }^{111} \mathrm{In} .{ }^{88}$ The research groups demonstrated that CNTs functionalized in this fashion underwent rapid renal clearance, with preferential accumulation ( $20 \%$ ) observed in muscle, skin, blood, and kidney, 30 minutes post-injection. This fell to $<1 \%$ after 3 hours and $<0.7 \%$ after 24 hours. The blood circulation half-life was $\sim 3.5$ hours. As seen with the noncovalently-functionalized 
CNTs, the blood circulation half-life was improved by increasing the degree of functionalization through the introduction of acid-induced surface defects, to which additional DTPA molecules were linked. ${ }^{152}$ The initial blood half-life $\left(t_{1 / 2}\right)$ of the CNTs with a high degree of functionalization measured $0.013 \mathrm{~h}^{-1}$, which demonstrates that the CNTs were distributed rapidly post-injection. The second phase blood half-life $\left(\mathrm{t}_{1 / 2 \beta}\right)$ measured $\sim 7$ hours. A similar study by Jain et al, ${ }^{8}$ employing 99mTc-labeled carboxylated MWNTs with different degrees of functionalization up to $\sim 3.4 \mu \mathrm{mol} / \mathrm{mg}$ carboxyl density, was evaluated. MWNTs were subjected to oxidation by acids for 1 hour, 2 hours, 4 hours, and 6 hours. Higher degrees of agglomeration of the CNTs was seen in 1-hour- and 2-hourtreated MWNTs, evident by the higher uptake and retention of these tubes, even after 24 hours, by the RES organs, such as the liver, spleen, and lungs. However, MWNTs oxidized for 4 hours and 6 hours, which also had considerable uptake by the RES organs, were slowly cleared in the span of 2-24 hours. This confirms the predominant role of the degree of functionalization in improving the density of coverage over CNTs, thus effectively shielding the entire surface of the rod. In the past, density and branching of the shielding materials were shown to be the most important parameters for determining blood circulation $\left(\mathrm{t}_{1 / 2 \beta}\right)$ and not length, chemistry, or architecture.

The ideal blood circulation half-life is application dependent. For imaging, which requires the particles to accumulate and achieve concentrations in the tissues followed by clearance, to produce adequate contrast, the optimum $t_{1 / 2}$ is 2-6 hours. In comparison, for therapeutic purposes, the optimal $\mathrm{t}_{1 / 2}$ is significantly longer to facilitate the repeated pooling of the particles in the tissue of interest. So far, the PEG-shielded CNT seems to be the polymer of choice for both imaging and therapeutics with its longer $t_{1 / 2}$, which is in comparison to the $\sim 7$-hour $t_{1 / 2}$ of covalently functionalized CNTs. However, some recent reports have shown that PEG-SWNT hybrids elicit concentration- and surface density-dependent cytotoxicity in PC12 cells, caused by oxidative stress. ${ }^{153}$ Another report suggests that PL-PEG moieties are responsible for complement activation by CNT, triggering the lectin pathway. ${ }^{154}$ Being one of the most extensively studied and reviewed molecules, PEG and its interactions with various particles are being elaborately characterized and reported. This augurs well for the biomedical community. Likewise, there is a need for alternative shielding agents that offer comparable, and sometimes better, efficacy after complexation with the particles. Pluronic surfactants such as F127 and F108 coated on MWNT have shown to afford better protection against nonspecific serum protein adsorption and anti-inflammatory processes. ${ }^{140,145}$ By effectively dispersing MWNTs, F108 passivates the CNT surface by forming a protective brush-like layer that is able to prevent profibrogenic responses in the macrophages through steric hindrance and, hence, have little cellular uptake. Another recently used strategy was to coat CNTs with a hydrophobic polystyrene polymer $(50 \% \mathrm{w} / \mathrm{w}){ }^{155}$ A considerable enhancement in the dispersion of polystyrene coated CNTs and a subsequent reduction in internalization by macrophages was reported. We also reported a novel strategy to coat SWNTs with gold to create "golden nanotubes", which benefits from the biocompatibility afforded by the surface gold plating and the complementary NIR absorption properties of the two materials. ${ }^{20}$ Highly specific localization of the golden nanotubes was observed in the lymphatic tissues 60 minutes post-injection, with persistence of the significant PA/PT signal up to 5 hours, indicating good biodistribution and blood residence time. However, just as was noticed in PEG, there is an increased risk of adverse reaction against synthetic materials. Therefore, natural biopolymers such as saccharides, proteins, and nucleic acids can be deployed to shield the CNTs. Our group has reported shielding the SWNTs with dextran sulfate to prevent opsonization by uniformly coating the SWNT surface in a facile manner to minimize exposure of the hydrophobic sidewall of the SWNT to the external milieu. ${ }^{39}$ We have also demonstrated that there was minimal internalization of dextran-SWNT constructs by human macrophages after preincubating with opsonins, $\mathrm{C} 3 \mathrm{~b}$, and Igs. ${ }^{25}$ Further, there is scope for employing biocompatible small molecules and proteins such as protein $\mathrm{A}$ and factor $\mathrm{H}$ complexes to SWNTs, to mimic common pathogens, such as Staphylococcus aureus, in evading the immune system.

\section{Conclusion}

CNTs represent a unique class of nanomaterials that have successfully bridged the gap between the biomedical and material sciences. A range of novel biomedical applications for CNTs has emerged in the last decade that showcases their potential for nanotheranostics. Several factors contribute to the stability and performance of CNTs in living systems, such as their biocompatibility, surface functionalization, biodistribution in tissues, and physicochemical characters including shape, size, and surface property, which were discussed in this review. Given their poor solubility in aqueous solvents and in their native aggregated state, processing strategies, such as coating with shielding agents or chemical treatment to modify the surface, is commonly practiced. Some of the widely used strategies include PEGylation, 
covalent functionalization with charged groups, and passivation through surfactants. Considerable improvement has been made in enhancing the blood circulation half-life and biodistribution of the hybrid CNT constructs. To date, PEGylated CNTs have demonstrated the maximal blood residence time ( $\sim 21$ hours) in comparison to covalently functionalized CNTs ( $\sim 7$ hours). The two routes of excretion have been distinctly delineated based on the shielding strategies: noncovalent, namely through the hepato-biliary system; and covalent, through the renal system.

However, there still is a need for alternative shielding strategies that can provide not only stealth character to the CNTs, but also additional "smart" functionality. In our opinion, novel shielding agents should provide "smart" functionality to the CNT constructs in addition to their usual property of increasing blood residence time. Additional conjugation sites for improving the stoichiometry and the loading density of ligands, their modularity to incorporate a wide variety of functionalization strategies, and their $\mathrm{pH}$ and oxygen sensitivity, are some of the desired properties to render CNT-based theranostic nanoagents as multimodal and semiautonomous. There, however, are many pertinent health-related challenges that need to be overcome before approaching the subject of real clinical translation, such as persistence in the tissues, elimination, and the long-term after-effects of CNTs. Interestingly, there are encouraging reports towards realizing the complete degradation and elimination of CNTs in vitro and in cellulo. Emerging evidence shows that catalytic enzymes such as horseradish peroxidase are able to degrade carboxylated CNTs in the presence of hydrogen peroxide. ${ }^{100}$ This would, however, require that the CNTs are eventually localized in the acidic environments of the endosomes over prolonged periods of time for complete degradation. Kagan et a ${ }^{99}$ have reported biodegradation of CNTs in cellulo, occurring in neutrophils with the help of a neutrophil enzyme, MPO. When aspirated into the lungs of mice, the degraded CNTs failed to elicit an inflammatory response, suggesting the benign nature of the degraded byproducts. Perhaps future coating strategies can exploit this unique biodegradation pathway available in the form of polymorphonuclear cells, and the associated catalytic enzymes, through selective uptake. This, however, should ideally occur during the clearance phase of CNT biodistribution in order to prevent early sequestration, degradation, and excretion before its intended purpose is realized. If semi-autonomous multimodal theranostic CNTbased nanoagents are envisaged, true hybrid constructs that take advantage of biological cues and natural systems should be designed. For example, the fates of foreign NPs and their hybrid nanoconstructs in vivo depend upon their physicobiochemical properties, including their size, shape, and surface chemistry. Some novel approaches have been reported to engineer NPs and their architectures with desirable size, shape, and functionality, including those based on the "nanotoolbox"-based programmable self-assembly approach developed by our group..$^{2,3,156,157}$ The capability of modulating the geometric configurations and surface characteristics suggests opportunities to overcome the hurdle, while considerably increasing blood circulation times as well as biocompatibility. ${ }^{128,129}$ There exist many biological particles, such as blood cells and pathogenic bacteria, that have evolved to evade macrophages by being transparent to the blood opsonins. Using lessons from nature, the design and construction of nanostructures with particular shapes and surface characteristics may permit us to avoid opsonization and premature clearance of nanotheranostics, ${ }^{2,3}$ enabling realization of NP-based theranostics and their translation into clinical practice. Finally, as the technology progresses, appealing new directions would arise that could transform the field of nanomedicine beyond those described in this review.

\section{Acknowledgments}

This work was supported in part by National Science Foundation grant numbers CMMI 1235100 and 0709121, ECCS1137948 and 1128660 , as well as by the Arkansas Biosciences Institute. The authors acknowledge Min Kim and Hee-Jeung Kim for their assistance in image processing.

\section{Disclosure}

The authors report no conflicts of interest in this work.

\section{References}

1. Buzea C, Pacheco II, Robbie K. Nanomaterials and nanoparticles: sources and toxicity. Biointerphases. 2007;2(4):MR17-MR71.

2. Kim JW, Galanzha EI, Zaharoff DA, Griffin RJ, Zharov VP. Nanotheranostics of circulating tumor cells, infections and other pathological features in vivo. Mol Pharm. 2013;10(3):813-830.

3. Kim JW, Deaton R. Molecular self-assembly of multifunctional nanoparticle composites with arbitrary shapes and functions: challenges and strategies. Particle and Particle Systems Characterization. 2013;30(2):117-132.

4. Davis ME, Chen ZG, Shin DM.Nanoparticle therapeutics: an emerging treatment modality for cancer. Nat Rev Drug Discov. 2008;7(9):771-782.

5. Sokolov I, Kievsky YY, Kaszpurenko JM. Self-assembly of ultrabright fluorescent silica particles. Small. 2007;3(3):419-423.

6. O'Connell MJ, Bachilo SM, Huffman CB, et al. Band gap fluorescence from individual single-walled carbon nanotubes. Science. 2002;297(5581):593-596.

7. Schipper ML, Nakayama-Ratchford N, Davis CR, et al. A pilot toxicology study of single-walled carbon nanotubes in a small sample of mice. Nat Nanotechnol. 2008;3(4):216-221. 
8. Jain S, Thakare VS, Das M, et al. Toxicity of multiwalled carbon nanotubes with end defects critically depends on their functionalization density. Chem Res Toxicol. 2011;24(11):2028-2039.

9. Deng XY, Jia G, Wang HF, et al. Translocation and fate of multi-walled carbon nanotubes in vivo. Carbon. 2007;45:1419-1424.

10. Mutlu GM, Budinger GR, Green AA, et al. Biocompatible nanoscale dispersion of single-walled carbon nanotubes minimizes in vivo pulmonary toxicity. Nano Lett. 2010;10(5):1664-1670.

11. Muller J, Delos M, Panin N, Rabolli V, Huaux F, Lison D. Absence of carcinogenic response to multiwall carbon nanotubes in a 2-year bioassay in the peritoneal cavity of the rat. Toxicol Sci. 2009;110(2): $442-448$.

12. Ali-Boucetta H, Nunes A, Sainz R, et al. Asbestos-like pathogenicity of long carbon nanotubes alleviated by chemical functionalization. Angew Chem Int Ed Engl. 2013;52(8):2274-2278.

13. Lacerda L, Ali-Boucetta H, Herrero MA, et al. Tissue histology and physiology following intravenous administration of different types of functionalized multiwalled carbon nanotubes. Nanomedicine (Lond). 2008;3(2):149-161.

14. Bianco A, Kostarelos K, Partidos CD, Prato M. Biomedical applications of functionalised carbon nanotubes. Chem Commun. 2005;7(5):571-577.

15. Pantarotto D, Briand JP, Prato M, Bianco A. Translocation of bioactive peptides across cell membranes by carbon nanotubes. Chem Commun (Camb). 2004;7(1):16-17.

16. de la Zerda A, Kim JW, Galanzha EI, Gambhir SS, Zharov VP. Advanced contrast nanoagents for photoacoustic molecular imaging, cytometry, blood test and photothermal theranostics. Contrast Media Mol Imaging. 2011;6(5):346-369.

17. Kam NW, Dai H. Carbon nanotubes as intracellular protein transporters: generality and biological functionality. J Am Chem Soc. 2005;127(16):6021-6026.

18. Panchapakesan B, Lu S, Sivakumar K, Taker K, Cesarone G, Wickstrom E. Single-wall carbon nanobomb agents for killing breast cancer cells Nanobiotechnology. 2005;1(2):133-139.

19. Zharov VP, Kim JW, Curiel DT, Everts M. Self-assembling nanoclusters in living systems: application for integrated photothermal nanodiagnostics and nanotherapy. Nanomedicine 2005;1(4):326-345.

20. Kim JW, Galanzha EI, Shashkov EV, Moon HM, Zharov VP. Golden carbon nanotubes as multimodal photoacoustic and photothermal highcontrast molecular agents. Nat Nanotechnol. 2009;4(10):688-694.

21. Iijima S. Helical microtubules of graphitic carbon. Nature. 1991;354: 56-58.

22. Baughman RH, Zakhidov AA, de Heer WA. Carbon nanotubes - the route toward applications. Science. 2002;297(5582):787-792.

23. König K. Multiphoton microscopy in life sciences. J Microsc. 2000; 200(Pt 2):83-104

24. Kim JW, Shashkov EV, Galanzha EI, Kotagiri N, Zharov VP. Photothermal antimicrobial nanotherapy and nanodiagnostics with self-assembling carbon nanotube clusters. Lasers Surg Med. 2007;39(7):622-634.

25. Kotagiri N, Lee JS, Kim JW. Selective pathogen targeting and macrophage evading carbon nanotubes through dextran sulfate coating and PEGylation for photothermal theranostics. J Biomed Nanotechnol. 2013;9(6):1008-1016

26. Moon HM, Kim JW. Carbon nanotube clusters as universal bacterial adsorbents and magnetic separation agents. Biotechnol Prog. 2010;26(1):179-185.

27. Galanzha EI, Shashkov E, Sarimollaoglu M, et al. In vivo magnetic enrichment, photoacoustic diagnosis, and photothermal purging of infected blood using multifunctional gold and magnetic nanoparticles. PLoS One. 2012;7(9):e45557.

28. Kang B, Chang S, Dai Y, Yu D, Chen D. Cell response to carbon nanotubes: size-dependent intracellular uptake mechanism and subcellular fate. Small. 2010;6(21):2362-2366.

29. Rawson FJ, Yeung CL, Jackson SK, Mendes PM. Tailoring 3D singlewalled carbon nanotubes anchored to indium tin oxide for natural cellular uptake and intracellular sensing. Nano Lett. 2013;13(1):1-8.
30. Lu Q, Moore JM, Huang G, et al. RNA polymer translocation with single-walled carbon nanotubes. Nano Lett. 2004;4(12):2473-2477.

31. Galanzha EI, Kim JW, Zharov VP. Nanotechnology-based molecular photoacoustic and photothermal flow cytometry platform for in-vivo detection and killing of circulating cancer stem cells. J Biophotonics. 2009;2(12):725-735.

32. Galanzha EI, Kokoska MS, Shashkov EV, Kim JW, Tuchin VV, Zharov VP. In vivo fiber-based multicolor photoacoustic detection and photothermal purging of metastasis in sentinel lymph nodes targeted by nanoparticles. J Biophotonics. 2009;2(8-9):528-539.

33. Galanzha EI, Shashkov EV, Kelly T, Kim JW, Yang L, Zharov VP. In vivo magnetic enrichment and multiplex photoacoustic detection of circulating tumour cells. Nat Nanotechnol. 2009;4(12):855-860.

34. Zharov VP, Galanzha EI, Shashkov EV, Kim JW, Khlebtsov NG, Tuchin VV. Photoacoustic flow cytometry: principle and application for real-time detection of circulating single nanoparticles, pathogens, and contrast dyes in vivo. J Biomed Opt. 2007;12(5):051503.

35. Yang WR, Thordarson P, Gooding JJ, Ringer SP, Braet F. Carbon nanotubes for biological and biomedical applications. Nanotechnology. 2007;18(41):412001.

36. Bahr JL, Tour JM. Covalent chemistry of single-wall carbon nanotubes. J Mater Chem. 2002;12:1952-1958.

37. Hirsch A. Functionalization of single-walled carbon nanotubes. Angew Chem Int Ed Engl. 2002;41(11):1853-1859.

38. Balavoine F, Schultz P, Richard C, Mallouh V, Ebbesen TW, Mioskowski C. Helical crystallization of proteins on carbon nanotubes: a first step towards the development of new biosensors. Agnew Chem Int Ed Engl. 1999;38(13):1912-1915.

39. Kotagiri N, Kim JW. Carbon nanotubes fed on "carbs": coating of single-walled carbon nanotubes by dextran sulfate. Macromol Biosci. 2010;10(3):231-238.

40. Star A, Steuerman DW, Heath JR, Stoddart JF. Starched carbon nanotubes. Angew Chem Int Ed Engl. 2002;41(14):2508-2512.

41. Zheng M, Jagota A, Semke ED, et al. DNA-assisted dispersion and separation of carbon nanotubes. Nat Mater. 2003;2(5):338-342.

42. Liu Z, Cai WB, He LN, et al. In vivo biodistribution and highly efficient tumour targeting of carbon nanotubes in mice. Nat Nanotechnol. 2007;2(1):47-52.

43. Chen RJ, Zhang Y, Wang D, Dai H. Noncovalent sidewall functionalization of single-walled carbon nanotubes for protein immobilization. J Am Chem Soc. 2001;123(16):3838-3839.

44. Kim JW, Kotagiri N, Kim JH, Deaton R. In situ fluorescence microscopy visualization and characterization of nanometer-scale carbon nanotubes labeled with 1-pyrenebutanoic acid, succinimidyl ester. Appl Phys Lett. 2006;88:213110.

45. Ju SY, Doll J, Sharma I, Papadimitrakopoulos F. Selection of carbon nanotubes with specific chiralities using helical assemblies of flavin mononucleotide. Nat Nanotechnol. 2008;3(6):356-362.

46. Narimatsu K, Niidome Y, Nakashima N. Pulsed-laser induced flocculation of carbon nanotubes solubilized by an anthracene-carrying polymer. Chem Phys Lett. 2006;429(4-6):488-491.

47. Fujigaya T, Morimoto T, Nakashima N. Isolated single-walled carbon nanotubes in a gel as a molecular reservoir and its application to controlled drug release triggered by near-IR laser irradiation. Soft Matter. 2011;7:2647-2652.

48. Niyogi S, Hamon MA, Hu H, et al. Chemistry of single-walled carbon nanotubes. Acc Chem Res. 2002;35(12):1105-1113.

49. Aránzazu Partearroyo M, Ostolaza H, Goñi FM, Barberá-Guillem E. Surfactant-induced cell toxicity and cell lysis. A study using B16 melanoma cells. Biochem Pharmacol. 1990;40(6):1323-1328.

50. Jos A, Pichardo S, Puerto M, Sánchez E, GriloA, CameánAM. Cytotoxicity of carboxylic acid functionalized single wall carbon nanotubes on the human intestinal cell line Caco-2. Toxicol In Vitro. 2009;23(8):1491-1496.

51. Pan BF, Cui DX, Xu P, et al. Cellular uptake enhancement of polyamidoamine dendrimer modified single walled carbon nanotubes. Journal of Biomedical and Pharmaceutical Engineering. 2007:13-16 
52. Wick P, Manser P, Limbach LK, et al. The degree and kind of agglomeration affect carbon nanotube cytotoxicity. Toxicol Lett. 2007;168(2):121-131.

53. Belyanskaya L, Manser P, Spohn P, Bruinink A, Wick P. The reliability and limits of the MTT reduction assay for carbon nanotubes-cell interaction. Carbon. 2007;45(13):2643-2648.

54. Wörle-Knirsch JM, Pulskamp K, Krug HF. Oops they did it again! Carbon nanotubes hoax scientists in viability assays. Nano Lett. 2006;6(6):1261-1268.

55. Movia D, Prina-Mello A, Bazou D, Volkov Y, Giordani S. Screening the cytotoxicity of single-walled carbon nanotubes using novel 3D tissue-mimetic models. ACS Nano. 2011;5(11):9278-9290.

56. Strano MS, Dyke CA, Usrey ML, et al. Electronic structure control of single-walled carbon nanotube functionalization. Science. 2003;301(5639):1519-1522.

57. Banerjee S, Wong SS. Selective metallic tube reactivity in the solutionphase osmylation of single-walled carbon nanotubes. J Am Chem Soc. 2004;126(7):2073-2081.

58. Vecitis CD, Zodrow KR, Kang S, Elimelech M. Electronic-structuredependent bacterial cytotoxicity of single-walled carbon nanotubes. ACS Nano. 2010;4(9):5471-5479.

59. Tong L, Liu Y, Dolash BD, et al. Label-free imaging of semiconducting and metallic carbon nanotubes in cells and mice using transient absorption microscopy. Nat Nanotechnol. 2012;7(1):56-61.

60. Huczko A, Lange H, Bystrzejewski M, et al. Pulmonary toxicity of 1-D nanocarbon materials. Fullerenes, Nanotubes, and Carbon Nanostructures. 2005;13:141-145.

61. Lam CW, James JT, McCluskey R, Hunter RL. Pulmonary toxicity of single-wall carbon nanotubes in mice 7 and 90 days after intratracheal instillation. Toxicol Sci. 2004;77(1):126-134.

62. Poland CA, Duffin R, Kinloch I, et al. Carbon nanotubes introduced into the abdominal cavity of mice show asbestos-like pathogenicity in a pilot study. Nat Nanotechnol. 2008;3(7):423-428.

63. Maynard AD, Baron PA, Foley M, Shvedova AA, Kisin ER, Castranova V. Exposure to carbon nanotube material: aerosol release during the handling of unrefined single-walled carbon nanotube material. J Toxicol Environ Health A. 2004;67(1):87-107.

64. Vivekchand SR, Jayakanth R, Govindaraj A, Rao CN. The problem of purifying single-walled carbon nanotubes. Small. 2005;1(10): 920-923.

65. Chen X, Tam UC, Czlapinski JL, et al. Interfacing carbon nanotubes with living cells. J Am Chem Soc. 2006;128(19):6292-6293.

66. Dumortier H, Lacotte S, Pastorin G, et al. Functionalized carbon nanotubes are non-cytotoxic and preserve the functionality of primary immune cells. Nano Lett. 2006;6(7):1522-1528.

67. Shi Kam NW, Jessop TC, Wender PA, Dai H. Nanotube molecular transporters: internalization of carbon nanotube-protein conjugates into Mammalian cells. J Am Chem Soc. 2004;126(22):6850-6851.

68. Pulskamp K, Diabaté S, Krug HF. Carbon nanotubes show no sign of acute toxicity but induce intracellular reactive oxygen species in dependence on contaminants. Toxicol Lett. 2007;168(1):58-74.

69. Cui D, Tian F, Ozkan CS, Wang M, Gao H. Effect of single wall carbon nanotubes on human HEK293 cells. Toxicol Lett. 2005;155(1) 73-85.

70. Kagan VE, Tyurina YY, Tyurin VA, et al. Direct and indirect effects of single walled carbon nanotubes on RAW 264.7 macrophages: role of iron. Toxicol Lett. 2006;165(1):88-100.

71. Shvedova AA, Castranova V, Kisin ER, et al. Exposure to carbon nanotube material: assessment of nanotube cytotoxicity using human keratinocyte cells. J Toxicol Environ Health A. 2003;66(20):1909-1926.

72. Isobe $H$, Tanaka $\mathrm{T}$, Maeda $\mathrm{R}$, et al. Preparation, purification, characterization, and cytotoxicity assessment of water-soluble, transitionmetal-free carbon nanotube aggregates. Angew Chem Int Ed Engl. 2006;45(40):6676-6680.

73. Ge C, Li Y, Yin JJ, et al. The contributions of metal impurities and tube structures to the toxicity of carbon nanotube materials. NPG Asia Materials. 2012;4:e32.
74. Choi JH, Nguyen FT, Barone PW, et al. Multimodal biomedical imaging with asymmetric single-walled carbon nanotube/iron oxide nanoparticle complexes. Nano Lett. 2007;7(4):861-867.

75. Al Faraj A, Cieslar K, Lacroix G, Gaillard S, Canet-Soulas E, Crémillieux Y. In vivo imaging of carbon nanotube biodistribution using magnetic resonance imaging. Nano Lett. 2009;9(3): $1023-1027$.

76. Al Faraj A, Fauvelle F, Luciani N, et al. In vivo biodistribution and biological impact of injected carbon nanotubes using magnetic resonance techniques. Int J Nanomedicine. 2011;6:351-361.

77. Bawa R. Nanoparticle-based therapeutics in humans: a survey. Nanotechnology Law and Business. 2008;5(2):135-155.

78. Monopoli MP, Aberg C, Salvati A, Dawson KA. Biomolecular coronas provide the biological identity of nanosized materials. Nat Nanotechnol. 2012;7(12):779-786.

79. Ge C, Du J, Zhao L, et al. Binding of blood proteins to carbon nanotubes reduces cytotoxicity. Proc Natl Acad Sci U S A. 2011;108(41): $16968-16973$.

80. Shannahan JH, Brown JM, Chen R, et al. Comparison of nanotubeprotein corona composition in cell culture media. Small. 2013;9(12): 2171-2181.

81. Cai X, Ramalingam R, Wong HS, et al. Characterization of carbon nanotube protein corona by using quantitative proteomics. Nanomedicine. 2013;9(5):583-593.

82. Ling WL, Biro A, Bally I, et al. Proteins of the innate immune system crystallize on carbon nanotubes but are not activated. ACS Nano. 2011;5(2):730-737.

83. Sacchetti C, Motamedchaboki K, Magrini A, et al. Surface polyethylene glycol conformation influences the protein corona of polyethylene glycol-modified single-walled carbon nanotubes: potential implications on biological performance. ACS Nano. 2013;7(3):1974-1989.

84. Doorley GW, Payne CK. Cellular binding of nanoparticles in the presence of serum proteins. Chem Commun (Camb). 2011;47(1):466-468.

85. Marano F, Hussain S, Rodrigues-Lima F, Baeza-Squiban A, Boland S. Nanoparticles: molecular targets and cell signalling. Arch Toxicol. 2011;85(7):733-741.

86. Wang H, Zhou W, Ho DL, et al. Dispersing single-walled carbon nanotubes with surfactants: a small angle neutron scattering study. Nano Lett. 2004;4(9):1789-1793.

87. Cherukuri P, Gannon CJ, Leeuw TK, et al. Mammalian pharmacokinetics of carbon nanotubes using intrinsic near-infrared fluorescence. Proc Natl Acad Sci U S A. 2006;103(50):18882-18886.

88. Singh R, Pantarotto D, Lacerda L, et al. Tissue biodistribution and blood clearance rates of intravenously administered carbon nanotube radiotracers. Proc Natl Acad Sci U S A. 2006;103(9):3357-3362.

89. McDevitt MR, Chattopadhyay D, Kappel BJ, et al. Tumor targeting with antibody-functionalized, radiolabeled carbon nanotubes. $\mathrm{J} \mathrm{Nucl}$ Med. 2007;48(7):1180-1189.

90. Kolhar P, Anselmo AC, Gupta V, et al. Using shape effects to target antibody-coated nanoparticles to lung and brain endothelium. Proc Natl Acad Sci U S A. 2013;110(26):10753-10758.

91. Albini A, Mussi V, Parodi A, et al. Interactions of single-wall carbon nanotubes with endothelial cells. Nanomedicine. 2010;6(2):277-288.

92. Hong G, Lee JC, Robinson JT, et al. Multifunctional in vivo vascular imaging using near-infrared II fluorescence. Nat Med. 2012;18(12): 1841-1846.

93. Braet F, Wisse E. Structural and functional aspects of liver sinusoidal endothelial cell fenestrae: a review. Comp Hepatol. 2002;1(1):1.

94. Lacerda L, Herrero MA, Venner K, Bianco A, Prato M, Kostarelos K. Carbon-nanotube shape and individualization critical for renal excretion. Small. 2008;4(8):1130-1132.

95. Ruggiero A, Villa $\mathrm{CH}$, Bander E, et al. Paradoxical glomerular filtration of carbon nanotubes. Proc Natl Acad Sci U S A. 2010;107(27) 12369-12374

96. Cheng SH, Li FC, Souris JS, et al. Visualizing dynamics of sub-hepatic distribution of nanoparticles using intravital multiphoton fluorescence microscopy. ACS Nano. 2012;6(5):4122-4131. 
97. Singh RP, Das M, Thakare V, Jain S. Functionalization density dependent toxicity of oxidized multiwalled carbon nanotubes in a murine macrophage cell line. Chem Res Toxicol. 2012;25(10):2127-2137.

98. Shvedova AA, Pietroiusti A, Fadeel B, Kagan VE. Mechanisms of carbon nanotube-induced toxicity: focus on oxidative stress. Toxicol Appl Pharmacol. 2012;261(2):121-133.

99. Kagan VE, Konduru NV, Feng W, et al. Carbon nanotubes degraded by neutrophil myeloperoxidase induce less pulmonary inflammation. Nat Nanotechnol. 2010;5(5):354-359.

100. Allen BL, Kichambare PD, Gou P, et al. Biodegradation of singlewalled carbon nanotubes through enzymatic catalysis. Nano Lett. 2008;8(11):3899-3903.

101. Allen BL, Kotchey GP, Chen Y, et al. Mechanistic investigations of horseradish peroxidase-catalyzed degradation of single-walled carbon nanotubes. J Am Chem Soc. 2009;131(47):17194-17205.

102. Shvedova AA, Kapralov AA, Feng WH, et al. Impaired clearance and enhanced pulmonary inflammatory/fibrotic response to carbon nanotubes in myeloperoxidase-deficient mice. PLoS One. 2012;7(3):e30923.

103. Nunes A, Bussy C, Gherardini L, et al. In vivo degradation of functionalized carbon nanotubes after stereotactic administration in the brain cortex. Nanomedicine (Lond). 2012;7(10):1485-1494.

104. Zhang L, Petersen EJ, Habteselassie MY, Mao L, Huang Q. Degradation of multiwall carbon nanotubes by bacteria. Environ Pollut. 2013;181:335-339.

105. Russier J, Ménard-Moyon C, Venturelli E, et al. Oxidative biodegradation of single- and multi-walled carbon nanotubes. Nanoscale. 2011;3(3):893-896.

106. Liu X, Hurt RH, Kane AB. Biodurability of single-walled carbon nanotubes depends on surface functionalization. Carbon NY. 2010;48(7): 1961-1969.

107. Zhao Y, Allen BL, Star A. Enzymatic degradation of multiwalled carbon nanotubes. J Phys Chem A. 2011;115(34):9536-9544.

108. Kamps JA, Scherphof GL. Receptor versus non-receptor mediated clearance of liposomes. Adv Drug Deliv Rev. 1998;32(1-2):81-97.

109. Kiwada H, Matsuo H, Harashima H. Identification of proteins mediating clearance of liposomes using a liver perfusion system. Adv Drug Deliv Rev. 1998;32(1-2):61-79.

110. Patel HM, Moghimi SM. Serum-mediated recognition of liposomes by phagocytic cells of the reticuloendothelial system - The concept of tissue specificity. Adv Drug Deliv Rev. 1998;32(1-2):45-60.

111. Sparrow CP, Parthasarathy S, Steinberg D. A macrophage receptor that recognizes oxidized low density lipoprotein but not acetylated low density lipoprotein. J Biol Chem. 1989;264(5):2599-2604.

112. Moghimi SM, Hunter AC, Murray JC. Long-circulating and target-specific nanoparticles: theory to practice. Pharmacol Rev. 2001;53(2):283-318.

113. Alexis F, Pridgen E, Molnar LK, Farokhzad OC. Factors affecting the clearance and biodistribution of polymeric nanoparticles. Mol Pharm. 2008;5(4):505-515.

114. Nagayama S, Ogawara K, Fukuoka Y, Higaki K, Kimura T. Time-dependent changes in opsonin amount associated on nanoparticles alter their hepatic uptake characteristics. Int J Pharm. 2007;342(1-2):215-221.

115. Champion JA, Mitragotri S. Role of target geometry in phagocytosis. Proc Natl Acad Sci U S A. 2006;103(13):4930-4934.

116. Harashima H, Sakata K, Funato K, Kiwada H. Enhanced hepatic uptake of liposomes through complement activation depending on the size of liposomes. Pharm Res. 1994;11(3):402-406.

117. Sund J, Alenius H, Vippola M, Savolainen K, Puustinen A. Proteomic characterization of engineered nanomaterial-protein interactions in relation to surface reactivity. ACS Nano. 2011;5(6):4300-4309.

118. Moghimi SM, Szebeni J. Stealth liposomes and long circulating nanoparticles: critical issues in pharmacokinetics, opsonization and proteinbinding properties. Prog Lipid Res. 2003;42(6):463-478.

119. Rudt S, Muller RH. In vitro phagocytosis assay of nano- and microparticles by chemiluminescence. III. Uptake of differently sized surface-modified particles, and its correlation to particle properties and in vivo distribution. Eur J Pharm Sci. 1993;1(1):31-39.
120. Shi X, von dem Bussche A, Hurt RH, Kane AB, Gao H. Cell entry of one-dimensional nanomaterials occurs by tip recognition and rotation. Nat Nanotechnol. 2011;6(11):714-719.

121. Allen TM. The use of glycolipids and hydrophilic polymers in avoiding rapid uptake of liposomes by the mononuclear phagocyte system. Adv Drug Deliv Rev. 1994;13(3):285-309.

122. Allen TM. A study of phospholipid interactions between high-density lipoproteins and small unilamellar vesicles. Biochim Biophys Acta. 1981;640(2):385-397.

123. Ilium L, Hunneyball IM, Davis SS. The effect of hydrophilic coatings on the uptake of colloidal particles by the liver and by peritoneal macrophages. Int J Pharm. 1986;29(1):53-65.

124. Jeon SI, Lee JH, Andrade JD, De Gennes PG. Protein-surface interactions in the presence of polyethylene oxide: I. Simplified theory. J Colloid Interface Sci. 1991;142(1):149-158.

125. Gabizon A, Papahadjopoulos D. The role of surface charge and hydrophilic groups on liposome clearance in vivo. Biochim Biophys Acta. 1992;1103(1):94-100.

126. Tabata Y, Ikada Y. Effect on the size and surface charge of polymer microspheres on their phagocytosis by macrophage. Biomaterials. 1988;9(4):356-362.

127. Tabata Y, Ikada Y. Phagocytosis of polymer microspheres by macrophages. Advances in Polymer Science. 1990;94:107-141.

128. Otsuka H, Nagasaki Y, Kataoka K. PEGylated nanoparticles for biological and pharmaceutical applications. Adv Drug Deliv Rev. 2003;55(3):403-419.

129. Coleman DL, Gregonis DE, Andrade JD. Blood-materials interactions: the minimum interfacial free energy and the optimum polar/apolar ratio hypotheses. J Biomed Mater Res. 1982;16(4): 381-398.

130. Antonsen KP, Hoffman AS. Water structure of PEG solutions by DSC measurements. In: Harris JM, editor. Poly(ethylene glycol) Chemistry: Biotechnical and Biomedical Applications. New York, NY: Springer; 1992:15-28.

131. Romberg B, Hennink WE, Storm G. Sheddable coatings for longcirculating nanoparticles. Pharm Res. 2008;25(1):55-71.

132. Gasser M, Rothen-Rutishauser B, Krug HF, et al. The adsorption of biomolecules to multi-walled carbon nanotubes is influenced by both pulmonary surfactant lipids and surface chemistry. J Nanobiotechnology. 2010;8:31.

133. Yamamoto Y, Nagasaki Y, Kato Y, Sugiyama Y, Kataoka K. Long-circulating poly(ethylene glycol)-poly(D,L-lactide) block copolymer micelles with modulated surface charge. J Control Release. 2001;77(1-2):27-38.

134. Yang M, Wada M, Zhang M, et al. A high poly(ethylene glycol) density on graphene nanomaterials reduces the detachment of lipid-poly(ethylene glycol) and macrophage uptake. Acta Biomater. 2013;9(1):4744-4753

135. Lunov O, Syrovets T, Loos C, et al. Differential uptake of functionalized polystyrene nanoparticles by human macrophages and a monocytic cell line. ACS Nano. 2011;5(3):1657-1669.

136. Liu F, Liu D. Serum independent liposome uptake by mouse liver. Biochim Biophys Acta. 1996;1278(1):5-11.

137. Kommareddy S, Amiji M. Biodistribution and pharmacokinetic analysis of long-circulating thiolated gelatin nanoparticles following systemic administration in breast cancer-bearing mice. J Pharm Sci. 2007;96(2):397-407.

138. Konduru NV, Tyurina YY, Feng W, et al. Phosphatidylserine targets single-walled carbon nanotubes to professional phagocytes in vitro and in vivo. PLoS One. 2009;4(2):e4398.

139. Wang X, Xia T, Ntim SA, et al. Dispersal state of multiwalled carbon nanotubes elicits profibrogenic cellular responses that correlate with fibrogenesis biomarkers and fibrosis in the murine lung. ACS Nano. 2011;5(12):9772-9787.

140. Wang X, Xia T, Duch MC, et al. Pluronic F108 coating decreases the lung fibrosis potential of multiwall carbon nanotubes by reducing lysosomal injury. Nano Lett. 2012;12(6):3050-3061. 
141. Bakota EL, Aulisa L, Tsyboulski DA, Weisman RB, Hartgerink JD. Multidomain peptides as single-walled carbon nanotube surfactants in cell culture. Biomacromolecules. 2009;10(8):2201-2206.

142. Liu D, Wang L, Wang Z, Cuschieri A. Different cellular response mechanisms contribute to the length-dependent cytotoxicity of multiwalled carbon nanotubes. Nanoscale Res Lett. 2012; 7(1):361.

143. Liu X, Tao H, Yang K, Zhang S, Lee ST, Liu Z. Optimization of surface chemistry on single-walled carbon nanotubes for in vivo photothermal ablation of tumors. Biomaterials. 2011;32(1):144-151.

144. Madani SY, Tan A, Dwek M, Seifalian AM. Functionalization of single-walled carbon nanotubes and their binding to cancer cells. Int J Nanomedicine. 2012;7:905-914.

145. Monteiro-Riviere NA, Inman AO, Wang YY, Nemanich RJ. Surfactant effects on carbon nanotube interactions with human keratinocytes. Nanomedicine. 2005;1(4):293-299.

146. Monteiro-Riviere NA, Nemanich RJ, Inman AO, Wang YY, Riviere JE. Multi-walled carbon nanotube interactions with human epidermal keratinocytes. Toxicol Lett. 2005;155(3):377-384.

147. Liu Z, Tabakman S, Welsher K, Dai H. Carbon nanotubes in biology and medicine: in vitro and in vivo detection, imaging and drug delivery. Nano Res. 2009;2(2):85-120.

148. Gasser M, Wick P, Clift MJ, et al. Pulmonary surfactant coating of multiwalled carbon nanotubes (MWCNTs) influences their oxidative and pro-inflammatory potential in vitro. Part Fibre Toxicol. 2012;9:17.

149. Vroman L, Adams AL, Fischer GC, Munoz PC. Interaction of high molecular weight kininogen, factor XII, and fibrinogen in plasma at interfaces. Blood. 1980;55(1):156-159.
150. Liu Z, Davis C, Cai W, He L, Chen X, Dai H. Circulation and long-term fate of functionalized, biocompatible single-walled carbon nanotubes in mice probed by Raman spectroscopy. Proc Natl Acad Sci U S A. 2008;105(5):1410-1415.

151. Kostarelos K, Bianco A, Prato M. Promises, facts and challenges for carbon nanotubes in imaging and therapeutics. Nat Nanotechnol. 2009;4(10):627-633.

152. Al-Jamal KT, Nunes A, Methven L, et al. Degree of chemical functionalization of carbon nanotubes determines tissue distribution and excretion profile. Angew Chem Int Ed Engl. 2012;51(26):6389-6393.

153. Zhang Y, Xu Y, Li Z, et al. Mechanistic toxicity evaluation of uncoated and PEGylated single-walled carbon nanotubes in neuronal PC12 cells ACS Nano. 2011;5(9):7020-7033.

154. Moghimi SM, Andersen AJ, Hashemi SH, et al. Complement activation cascade triggered by PEG-PL engineered nanomedicines and carbon nanotubes: the challenges ahead. $J$ Control Release. 2010;146(2): 175-181.

155. Tabet L, Bussy C, Setyan A, et al. Coating carbon nanotubes with a polystyrene-based polymer protects against pulmonary toxicity. Part Fibre Toxicol. 2011;8:3.

156. Kim JW, Kim JH, Deaton R. Programmable construction of nanostructures: assembly of nanostructures with various nanocomponents. IEEE Nanotechnol Mag. 2012;6(1):19-23.

157. Kim JW, Kim JH, Deaton R. DNA-linked nanoparticle building blocks for programmable matter. Angew Chem Int Ed Engl. 2011;50(39): 9185-9190.
International Journal of Nanomedicine

\section{Publish your work in this journal}

The International Journal of Nanomedicine is an international, peerreviewed journal focusing on the application of nanotechnology in diagnostics, therapeutics, and drug delivery systems throughout the biomedical field. This journal is indexed on PubMed Central,

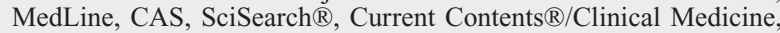

\section{Dovepress}

Journal Citation Reports/Science Edition, EMBase, Scopus and the Elsevier Bibliographic databases. The manuscript management system is completely online and includes a very quick and fair peer-review system, which is all easy to use. Visit http://www.dovepress.com/ testimonials.php to read real quotes from published authors. 